\title{
Guaranteeing Timely Response to Changes of Monitored Objects by Assigning Deadlines and Periods to Tasks
}

\author{
QUAN ZHOU, GUOHUI LI, QI CHEN, and JIANJUN LI, Huazhong University of Science and \\ Technology, China
}

\begin{abstract}
Timely response to changes of monitored objects is the key to ensuring the safety and reliability of cyberphysical systems (CPSs). There are two kinds of tasks in CPSs: update tasks and control tasks. Update tasks are responsible for updating the data in the system based on the state of the objects they monitor. Control tasks are responsible for making decisions based on the data in the system. The response time of the system to the change of a monitored object consists of two parts: the time taken by update tasks to reflect the change to the system, and the time taken by control tasks to make decisions according to the data in the system. Deadlines and periods of update tasks and control tasks directly affect the response time. Reasonable deadline and period assignment is the key to ensuring timely response to the changes of monitored objects. In this paper, we study the deadline and period assignment in CPSs. To the best of our knowledge, all existing work only focuses on the deadline and period assignment for update tasks with the goal of ensuring the freshness of the data in CPSs, and this is the first study focusing on the deadline and period assignment for both update tasks and control tasks with the goal of ensuring timely response to the changes of monitored objects. A new problem about response time control and system workload control is defined in this paper. Two deadline and period assignment methods are proposed to solve the defined problem. All the proposed methods can be used in the CPSs adopting the earliest deadline first (EDF) scheduling method. Experiments with randomly generated tasks are conducted to evaluate the performance of the proposed methods in terms of acceptance ratio and execution efficiency.
\end{abstract}

CCS Concepts: • Computer systems organization $\rightarrow$ Real-time operating systems; Embedded software;

Additional Key Words and Phrases: Deadline and period assignment, hybrid tasks, timely response, EDF scheduling, CPSs

\section{ACM Reference format:}

Quan Zhou, Guohui Li, Qi Chen, and Jianjun Li. 2021. Guaranteeing Timely Response to Changes of Monitored Objects by Assigning Deadlines and Periods to Tasks. ACM Trans. Embedd. Comput. Syst. 20, 5s, Article 96 (September 2021), 22 pages.

https://doi.org/10.1145/3477027

This article appears as part of the ESWEEK-TECS special issue and was presented in the International Conference on Embedded Software (EMSOFT), 2021.

The work was partially supported by the National Natural Science Foundation of China under Grant No. 61802137, and Hubei Provincial Natural Science Foundation of China under Grant No. 2018CFB204.

Authors' address: Q. Zhou, G. Li, Q. Chen (corresponding author), and J. Li, Huazhong University of Science and Technology, 1037 Luoyu Rd, Wuhan, China; emails: \{quanzhou, guohuili, chenqijason, jianjunli\}@hust.edu.cn.

Permission to make digital or hard copies of all or part of this work for personal or classroom use is granted without fee provided that copies are not made or distributed for profit or commercial advantage and that copies bear this notice and the full citation on the first page. Copyrights for components of this work owned by others than ACM must be honored. Abstracting with credit is permitted. To copy otherwise, or republish, to post on servers or to redistribute to lists, requires prior specific permission and/or a fee. Request permissions from permissions@acm.org.

(-) 2021 Association for Computing Machinery.

1539-9087/2021/09-ART96 $\$ 15.00$

https://doi.org/10.1145/3477027 


\section{INTRODUCTION}

Cyber-physical systems (CPSs) have been widely used in many fields, such as health monitoring, industry control and vehicle control [10, 19, 24, 32, 34]. In some practical applications, CPSs need to monitor external objects and respond to every change of every monitored object in a timely manner $[6,7]$. For example, the driverless system needs to complete the braking operation in time once an obstacle suddenly appears in front, and the boiler control system needs to monitor the temperature of the high-pressure boiler in real time and close the heating valve within $100 \mathrm{~ms}$ once the temperature is too high.

In the CPS, there are many data items used to record the state of the monitored objects. Once the state of a monitored object changes, the system first updates the data item recording the state of this object, and then makes decisions based on the latest data in the system. So, the response time of the system to each change of each monitored object can be divided into two parts: the time taken to reflect the change to the system, and the time taken to make decisions based on the data in the system. Obviously, all these two parts are important to ensure that the system can respond to the changes of monitored objects in timely manner. However, all existing work $[11,14,15,18,30,31$, $36-38,40]$ on deadline and period assignment only focuses on how to ensure the freshness of the data in the system. More specifically, all existing work assumes that each state of each monitored object has a validity time interval. The data that records a state of a monitored object remains valid until the validity time interval of this state expires. All existing deadline and period assignment methods are designed to ensure that all data in the system is valid at any moment. This means all existing work only focuses on the time taken to reflect the changes of monitored objects to the system, but does not consider the response time of the system to the changes of monitored objects. Since timely response to the changes of monitored objects is the key to ensuring the safety and reliability of CPSs, it is necessary to design an effective method to ensure the timely response.

There are two kinds of tasks in CPSs: update tasks and control tasks. Update tasks are responsible for monitoring external objects and updating the data in the system. Control tasks are responsible for reading data from the system and making decisions based on the data they obtain. The attributes of update tasks affect the time taken to reflect the changes of monitored objects to the system. The attributes of control tasks affect the time taken to make decisions based on the data in the system. Since the response time of the system to each change of each monitored object can be divided into two parts, the time taken to reflect this change to the system and the time taken to make decisions, reasonable deadline and period assignment for update tasks and control tasks is the key to ensuring timely response (to the changes of monitored objects).

Since the tasks in CPSs are usually real-time, CPSs usually use real-time scheduling methods to schedule the update tasks and control tasks in CPSs. The fixed priority (FP) scheduling and the earliest deadline first (EDF) scheduling are two kinds of representative real-time scheduling methods. Under FP scheduling, each task has a fixed priority. The jobs generated by each task inherit the priority of this task. Under EDF scheduling, the priorities of tasks are not fixed, while each job has a fixed priority that is inversely proportional to its deadline. In this paper, we study the deadline and period assignment method for tasks (including update tasks and control tasks) under EDF scheduling with the goal of ensuring timely response to the changes of monitored objects.

A new problem focusing on response time control and system workload control is defined in this paper. Two deadline and period assignment methods named expended co-scheduling (ECS) and harmonic co-scheduling (HCS) are proposed to solve this problem. The ECS method regards change-reflecting and decision-making as two independent processes. For limiting the response time to the changes of monitored objects, ECS limits the time cost in the first process by setting reasonable deadlines and periods for update tasks, and limits the time cost in the second process by 
setting reasonable deadlines and periods for control tasks. Unlike ECS, the HCS method does not limit the time cost in each process, but enables the system to complete the response to each change of each monitored object before the absolute deadline of a specific job, where the specific job refers to the first update job that captures this change. Theoretical analysis proves the effectiveness of the proposed methods. Experimental results show the performance of the proposed methods in terms of acceptance ratio and execution efficiency.

The rest of this paper is organized as follows: Section 2 reviews the previous work on deadline and period assignment. Section 3 introduces the notations and defines the problem studied in this paper. Section 4 proposes two deadline and period assignment methods to solve the defined problem. Section 5 presents the performance evaluation of the proposed methods, followed by the conclusion in Section 6.

\section{RELATED WORK}

This section reviews previous work on maintaining the freshness of the data in real-time database systems (RTDBSs) [8, 10, 21-23, 27, 40] and preserving freshness constraints in control networks.

\subsection{Related Work on Maintaining the Freshness of the Data in RTBDSs}

Kuo and Mok [26] investigated real-time data semantics and proposed the SSP protocol. Song and Liu [35] studied the performance of the two-phase locking and the optimistic algorithm in terms of maintaining temporal consistency of shared data in hard real-time systems with periodic tasks. Xiong et al. [41] introduced the concept of data-deadline and proposed a scheduling algorithm based on the forced-wait and similarity calculation techniques. The proposed method can maintain the temporal consistency of the data in real-time database systems. Gustafsson and Hansson [11] studied how to guarantee the freshness of the data in soft real-time systems, and proposed the ODDFT method. Gustafsson and Hansson [10] proposed the ODTB method that can reduce the CPU utilization by skipping some unnecessary updates for data. All above work assumes that the deadlines and periods of transactions have been assigned by the system, and thus only focuses on the task scheduling for maintaining the temporal consistency and the freshness of data.

Multiple task scheduling methods considering the deadline and period assignment are proposed in recent two decades. Ho et al. [20] proposed the $H H$ method that always sets the same value to the deadline and period of every update transaction. Xiong and Ramamritham [40] considered the environment in which update transactions generate jobs in their fixed periods, and proposed the $M L$ method. Xiong et al. [39] proposed the DS-FP method which supports the sporadic transaction model and can reduce the processor workload by deferring the sampling times of update transactions. Han et al. [14] proposed the DS-EDF method which extends DS-FP to the EDF scheduling environment. Jha et al. [21] gave some formulas that can get the maximal gaps among data reading time, and proposed a period deriving method which can guarantee the mutual temporal consistency of real-time data objects. Han et al. [15] focused on the problem of scheduling mode conversion, and proposed two effective switch point calculation methods. Zhu et al. [43] studied the deadline and period assignment for update transactions under the EDF scheduling, and proposed a linear programming-based method. All the above methods can only be used in the environments with only update transactions or only control transactions. To solve the co-scheduling problem of hybrid transactions, Han et al. [18] proposed the AEDF-Co method that can maximize the quality of data $(\mathrm{QoD})$ under the premise that all control tasks are schedulable. Wang et al. [37] proposed the PCS method that can adjust the relative priority between update transactions and control transactions and maximize the QoD while ensuring the schedulability of control tasks. Han et al. [17] proposed the Co-LALF method that can maximize the quality of 
the data accessed by currently executing control tasks. The above three methods can ensure the schedulability of control transactions, but update transactions may miss their deadlines. Li et al. [28] proposed the $\min D^{*}$ method that can assign deadlines and periods for update transactions under the guarantee that all tasks are schedulable under EDF.

Different from the above mentioned work, we focus on how to assign deadlines and periods for both update tasks and control tasks, such that all tasks are schedulable and the system can respond to each change of each monitored object in time.

\subsection{Related Work on Preserving Freshness Constraints in Control Networks}

Data freshness is used as a data quality metric in cyber-physical systems [16, 29]. [4] and [33] propose a comprehensive overview of freshness as a data quality metric and also a framework for analysis of freshness.

There are many algorithms to minimize the update workload while maintaining real-time data freshness. A simple method Half-Half $(\mathrm{HH})$ scheme is used in [25], where the update period of a real-time data object is set to be half the validity period of the object. To further reduce the update workload, the More-Less (ML) scheme was proposed in [36], which uses Deadline Monotonic (DM) to schedule periodic update transactions. Both of the above schemes are applicable to the periodic task model. However, [13] presents Deferrable Scheduling for Fixed-Priority transactions (DS-FP) to maintain data freshness, which adopts an aperiodic task model. The DS-FP update transaction jobs by deferring the sampling time of a transaction job as much as possible to reduce the workload. [16] uses different scheduling policies in different modes to maintain data freshness in the presence of mode changes in cyber physical systems. [29] explores a dynamic priority scheduling method to keep data freshness considering jitter, which calculates the period and deadline of update transaction to reduce workload.

In the practical application of embedded system, in addition to individual timing requirements on the response times of the tasks, the end-to-end functionality of the task chains are often required. Feiertag et al. propose a method to compute the different end-to-end delays of multi-rate cause-effect chains, where detailed scheduling information is available [5]. Becker et al. [1,2] propose a general framework for calculating the data age of cause-effect chains, which is specific to single processor system without considering scheduling strategy. [3] solve the problem of calculating the end-to-end delay bound on multi-core, and improved the result of [2]. Recently, [9] proposes an analytical framework which derives the best period to bound data freshness of task chains, remaining completely agnostic to the scheduling strategy.

\section{NOTATIONS AND PROBLEM DEFINITION}

\subsection{Model and Notations}

In this work, we consider a uni-processor CPS with $n$ monitored objects, $n$ update tasks and $x$ control tasks. We use $O=\left\{o_{1}, o_{2}, \ldots, o_{n}\right\}$ to denote the set consisting of all monitored objects, and use $\mathcal{D}=\left\{d_{1}, d_{2}, \ldots, d_{n}\right\}$ to denote the data set in the system, where $d_{i}$ is the data that records the state of $o_{i} . \mathcal{T}^{u}=\left\{\tau_{1}^{u}, \tau_{2}^{u}, \ldots, \tau_{n}^{u}\right\}$ and $\mathcal{T}^{c}=\left\{\tau_{1}^{c}, \tau_{2}^{c}, \ldots, \tau_{x}^{c}\right\}$ are used to denote the update task set and the control task set, respectively. $\mathcal{T}=\mathcal{T}^{u} \cup \mathcal{T}^{c}$ is the hybrid task set consisting of all tasks in the system.

Each monitored object $o_{i}$ has a valid response time $V_{i}$. Once the state of $o_{i}$ changes, the system needs to first update $d_{i}$ based on the new state of $o_{i}$, and then make decisions based on the new $d_{i}$. The whole process from the change of $o_{i}$ to the decision making based on the new $d_{i}$ needs to be completed within $V_{i}$ time units. 
Each update task $\tau_{i}^{u}$ can be characterized by a 3-tuple: $\left\langle C_{i}^{u}, D_{i}^{u}, T_{i}^{u}\right\rangle$, where $C_{i}^{u}$ is the worst case execution time (WCET), $D_{i}^{u}$ is the relative deadline and $T_{i}^{u}$ is the period. There is a one-to-one correspondence between update tasks and monitored objects (also data items in $\mathcal{D}$ ), that is,

- Each update task $\tau_{i}$ is responsible for monitoring only one object $o_{i}$ and updating only one data item $d_{i}$;

- Each object $o_{i}$ is monitored by only one update task $\tau_{i}$;

- Each data item $d_{i}$ is updated by only one update task $\tau_{i}$.

Each update task $\tau_{i}^{u}$ can generate a finite or infinite stream of jobs. We use $J_{i, j}^{u}$ to denote the $j$-th job of $\tau_{i}^{u}$. Each update job $J_{i, j}^{u}$ has a release time $r_{i, j}^{u}$, a start time $s_{i, j}^{u}$, an execution finish time $f_{i, j}^{u}$ and an absolute deadline $d_{i, j}^{u}\left(d_{i, j}^{u}=r_{i, j}^{u}+D_{i}^{u}\right)$. The release times of any two consecutively released jobs $J_{i, j}^{u}$ and $J_{i, j+1}^{u}, r_{i, j}^{u}$ and $r_{i, j+1}^{u}$, satisfy $r_{i, j+1}^{u}=r_{i, j}^{u}+T_{i}^{u}$. We assume that each update job $J_{i, j}^{u}$ samples $o_{i}$ at $r_{i, j}^{u}$ and completes the update of $d_{i}$ at $f_{i, j}^{u}$. An update job $J_{i, j}^{u}$ is schedulable if $f_{i, j}^{u} \leq d_{i, j}^{u}$. An update task $\tau_{i}^{u}$ is schedulable if all jobs generated by $\tau_{i}^{u}$ are schedulable.

Each control task $\tau_{i}^{c}$ can be characterized by a 4-tuple: $\left\langle C_{i}^{c}, D_{i}^{c}, T_{i}^{c}, A_{i}^{c}\right\rangle$, where $C_{i}^{c}$ is the WCET, $D_{i}^{c}$ is the relative deadline, $T_{i}^{c}$ is the period and $A_{i}^{c}$ is the data set consists of all data items accessed by $\tau_{i}^{c}$. A control task may access multiple data items, and a data item may be accessed by multiple control tasks. We use $\mathcal{T}_{i}^{c}$ to denote the task set consisting of all control tasks accessing $d_{i}$. Each control task $\tau_{i}^{c}$ can generate a finite or infinite stream of jobs. $J_{i, j}^{c}$ is used to denote the $j$-th job of $\tau_{i}^{c}$. Each control job $J_{i, j}^{c}$ has a release time $r_{i, j}^{c}$, a start time $s_{i, j}^{c}$, an execution finish time $f_{i, j}^{c}$ and an absolute deadline $d_{i, j}^{c}\left(d_{i, j}^{c}=r_{i, j}^{c}+D_{i}^{c}\right)$. Similar to update jobs, we assume that the release times of any two consecutively released jobs $J_{i, j}^{c}$ and $J_{i, j+1}^{c}, r_{i, j}^{c}$ and $r_{i, j+1}^{c}$, satisfy $r_{i, j+1}^{c}=r_{i, j}^{c}+T_{i}^{c}$. Moreover, for each control job $J_{i, j}^{c}$, the moment when it reads data cannot be earlier than $r_{i, j}^{c}$, and the moment when it completes the decision making cannot be later than $f_{i, j}^{c}$. A control job $J_{i, j}^{c}$ is schedulable if $f_{i, j}^{c} \leq d_{i, j}^{c}$. A control task $\tau_{i}^{c}$ is schedulable if all jobs generated by $\tau_{i}^{c}$ are schedulable.

We use $U_{i}^{u}=C_{i}^{u} / T_{i}^{u}$ and $U_{i}^{c}=C_{i}^{c} / T_{i}^{c}$ to denote the utilizations of $\tau_{i}^{u}$ and $\tau_{i}^{c}$, respectively. $U_{\text {total }}=\sum_{1 \leq i \leq n} U_{i}^{u}+\sum_{1 \leq i \leq x} U_{i}^{c}$ is the total utilization of $\mathcal{T}$.

\subsection{Harmonic Tasks and the $S_{r}$ Algorithm}

The 2-harmonic task set is a concept that will be used in this paper. We call a task set $\mathcal{T}$ a 2harmonic task if the period of any task is $2^{y}\left(y \in 0 \cup N^{+}\right)$times of other task periods which are not larger than this period. For example, the task set consisting of four tasks with periods 3, 6, 6, 24 is a 2-harmonic task set. The $S_{r}$ algorithm [12] is an effective method that can convert any task set into a 2-harmonic task set. Given a task set $\mathcal{T}=\left\{\tau_{1}, \tau_{2}, \ldots, \tau_{z}\right\}$, the $S_{r}$ algorithm processes $\mathcal{T}$ as follows: (1) for each task $\tau_{i}$ in $\mathcal{T}, S_{r}$ calculates a special value $\gamma_{i}$ by $\gamma_{i}=T_{i} / 2^{\left\lceil\log _{2}\left(T_{i} / T^{\prime}\right)\right\rceil}$, where $T_{i}$ is the period of $\tau_{i}$ and $T^{\prime}$ is the shortest period of all tasks; (2) based on each special value $\gamma_{i}, S_{r}$ converts $\mathcal{T}$ into a 2-harmonic task set by changing the period of each task $\tau_{k}$ by $T_{k}=\gamma_{i} * 2^{\left\lfloor\log _{2}\left(T_{k} / \gamma_{i}\right)\right\rfloor}$; (3) $S_{r}$ calculates the total utilization of each 2-harmonic task set, and takes the 2-harmonic task set with the minimum total utilization as the final 2-harmonic task set. The notations used in this paper are shown in Table 1.

\subsection{Problem Definition}

The deadline and period calculation (DPC) is an existing problem similar to the problem studied in this paper. DPC assumes that each sample value of each monitored object $o_{i}$ is valid only in a special time interval with length $V_{i}^{u}$. To ensure the freshness of the data in the system, the deadline and period of each update task $\tau_{i}^{u}$ need to satisfy $D_{i}^{u}+T_{i}^{u} \leq V_{i}^{u}$. The DPC problem was first raised in the RTDBS field, and its specific definition is as follows. 
Table 1. List of Notations

\begin{tabular}{cccc}
\hline Notations & Physical Meanings & Notations & Physical Meanings \\
\hline$n$ & number of update tasks & $x$ & number of control tasks \\
$O$ & monitored object set & $o_{i}$ & $i$-th object in $O$ \\
$\mathcal{D}$ & data set in the system & $d_{i}$ & the data corresponding to $o_{i}$ \\
$\mathcal{T}^{c}$ & control task set & $\mathcal{T}^{u}$ & update task set \\
$\mathcal{T}$ & $\mathcal{T}=\mathcal{T}^{u} \cup \mathcal{T}^{c}$ & $V_{i}$ & valid response time of $o_{i}$ \\
$\tau_{i}^{u}$ & update task corresponding to $d_{i}$ & $C_{i}^{u}$ & WCET of $\tau_{i}^{u}$ \\
$D_{i}^{u}$ & deadline of $\tau_{i}^{u}$ & $T_{i}^{u}$ & period of $\tau_{i}^{u}$ \\
$J_{i, j}^{u}$ & $j$-th job of $\tau_{i}^{u}$ & $r_{i, j}^{u}$ & release time of $J_{i, j}^{u}$ \\
$s_{i, j}^{u}$ & start time of $J_{i, j}^{u}$ & $f_{i, j}^{u}$ & execution finish time of $J_{i, j}^{u}$ \\
$d_{i, j}^{u}$ & absolute deadline of $J_{i, j}^{u}$ & $\tau_{i}^{c}$ & $i$-th task in $\mathcal{T}^{c}$ \\
$C_{i}^{c}$ & WCET of $\tau_{i}^{c}$ & $D_{i}^{c}$ & deadline of $\tau_{i}^{c}$ \\
$T_{i}^{c}$ & period of $\tau_{i}^{c}$ & $A_{i}^{c}$ & data set accessed by $\tau_{i}^{c}$ \\
$\mathcal{T}_{i}^{c}$ & control tasks reading $d_{i}$ & $J_{i, j}^{c}$ & $j$-job of $\tau_{i}^{c}$ \\
$r_{i, j}^{c}$ & release time of $J_{i, j}^{c}$ & $s_{i, j}^{c}$ & start time of $J_{i, j}^{c}$ \\
$f_{i, j}^{c}$ & execution finish time of $J_{i, j}^{c}$ & $d_{i, j}^{c}$ & absolute deadline of $J_{i, j}^{c}$ \\
$U_{i}^{u}$ & utilization $\tau_{i}^{u}$ & $U_{i}^{c}$ & utilization $\tau_{i}^{c}$ \\
$U_{\text {total }}^{c}$ & total utilization $\mathcal{T}$ & & \\
\hline
\end{tabular}

Deadline and period calculation problem [28]. Given a transaction set $\mathcal{T}$ which consists of update transaction set $\mathcal{T}^{u}$ and control transaction set $\mathcal{T}^{c}$, derive deadlines and periods for the update transactions in $\mathcal{T}^{u}$, such that,

1. $\mathcal{T}$ is schedulable under EDF.

2. The total workload of $\mathcal{T}, U_{\text {total }}$, is minimized.

3. All data in the database is valid at every moment.

Obviously, the DPC problem focuses on the time interval between two adjacent data updates. Unlike the DPC problem, the problem studied in this work focuses on the time interval between the moment when a monitored object changes and the moment when the corresponding decisions are completed. In this paper, we call this time interval the response time of the system to the changes of monitored objects. In addition, DPC assumes that the deadlines and periods of control transactions are known, while we assume that the deadlines and periods of both control tasks and update tasks are undetermined until the deadline and period assignment is completed. The definition of the problem studied in this paper is as follows.

Deadline and period calculation under response time constraints (DPC-RTC). Given a monitored object set $O$ and a hybrid task set $\mathcal{T}$ consisting of a control task set $\mathcal{T}^{c}$ and an update task set $\mathcal{T}^{u}$, derive deadlines and periods for tasks in $\mathcal{T}$, such that:

1. $\mathcal{T}$ is schedulable under EDF.

2. The total workload of $\mathcal{T}$ is minimized.

3. The response time of the system to each change of each monitored object $o_{i}$ does not exceed $V_{i}$.

\section{METHODS TO SOLVE THE DPC-RTC PROBLEM}

In this section, we propose two deadline and period assignment methods, ECS and HCS, to solve the DPC-RTC problem. Before showing ECS and HCS, we first consider a simple system environment and propose two simplified versions of the above two methods, simplified ECS (ECS-S) and simplified HCS (HCS-S). 


\subsection{Methods in Simple Environments}

We first consider a simple environment in which each control task $\tau_{i}^{c}$ accesses only one data item $d_{i}$ and each data item $d_{i}$ is accessed by only one control task $\tau_{i}^{c}$. In this environment, for each data item $d_{i}$, there is only one task pair $\left\langle\tau_{i}^{u}, \tau_{i}^{c}>\right.$ that consists of an update task and a control task and satisfies: (1) the update task is responsible for updating this data item; and (2) the control task needs to read this data item. For simplicity of expression, we call this kind of task pair the task pair corresponding to $d_{i}$.

As described in Section 1, the response process of the system to each change of each monitored object can be divided into two stages: reflecting the change to the system and making decisions based on the data in the system. The design idea of ECS-S (also ECS) is to limit the time cost in each of the above two stages by assigning reasonable deadlines and periods to update tasks and control tasks. Before showing the ECS-S method, we first give a theorem that explains the relation between the response time and the attributes of update tasks and control tasks.

Theorem 4.1. Let $V_{i}^{u}=D_{i}^{u}+T_{i}^{u}$ and $V_{i}^{c}=D_{i}^{c}+T_{i}^{c}$, if all tasks are schedulable under EDF, the response time to each change of each monitored object $o_{i}$ cannot exceed $V_{i}^{u}+V_{i}^{c}-\min \left\{C_{i}^{u}, C_{i}^{c}\right\}$.

Proof. Consider an arbitrary monitored object $o_{i}$ and use $t$ to represent an arbitrary change time of $o_{i}$. Since $d_{i}$ is the data item that records the state of $o_{i}$ and $\left\langle\tau_{i}^{u}, \tau_{i}^{c}\right\rangle$ is the only task pair corresponding to $d_{i}$, the response to the change of $o_{i}$ at $t$ needs to be implemented by executing $\tau_{i}^{u}$ and $\tau_{i}^{c}$. Use $J_{i, j}^{u}$ to represent the first job that captures this change, and use $J_{i, k}^{c}$ to represent the first job that captures the new $d_{i}$ updated by $J_{i, j}^{u}$. Since the decision-making by $J_{i, k}^{c}$ will be completed before the execution finish time of $J_{i, k}^{c}$ (i.e., before $f_{i, k}^{c}$ ), $f_{i, k}^{c}-t$ is an upper bound of the response time to this change. Since $o_{i}$ is an arbitrary monitored object and $t$ is an arbitrary change time of $o_{i}$, the theorem holds if $f_{i, k}^{c}-t \leq V_{i}^{u}+V_{i}^{c}-\min \left\{C_{i}^{u}, C_{i}^{c}\right\}$.

Note that $f_{i, k}^{c}-t$ can be converted into $\left(d_{i, j}^{u}-t\right)+\left(f_{i, k}^{c}-d_{i, j}^{u}\right)$. We first prove that $d_{i, j}^{u}-t<V_{i}^{u}$. Since update jobs sample monitored objects at their release times and $J_{i, j}^{u}$ is the first job that captures the change at $t, J_{i, j-1}^{u}$ does not capture this change and its release time $r_{i, j-1}^{u}$ must be earlier than $t$ (i.e., $\left.r_{i, j-1}^{u}<t\right)$. Therefore, we can get the following equality:

$$
\begin{aligned}
d_{i, j}^{u}-t & =D_{i}^{u}+r_{i, j}^{u}-t \\
& =D_{i}^{u}+T_{i}^{u}+r_{i, j-1}^{u}-t \\
& =V_{i}^{u}+r_{i, j-1}^{u}-t \\
& <V_{i}^{u}
\end{aligned}
$$

Next, we prove that $f_{i, k}^{c}-d_{i, j}^{u} \leq V_{i}^{c}-\min \left\{C_{i}^{u}, C_{i}^{c}\right\}$. Since all tasks are schedulable, the attributes of $\tau_{i}^{u}$ and $\tau_{i}^{c}$ satisfy $C_{i}^{u} \leq D_{i}^{u}$ and $C_{i}^{c} \leq D_{i}^{c}$, and the attributes of $J_{i, j}^{u}$ and $J_{i, k}^{c}$ satisfy $f_{i, j}^{u} \leq d_{i, j}^{u}$ and $f_{i, k}^{c} \leq d_{i, k}^{c}$. Therefore, we have the following equalities:

$$
\begin{gathered}
f_{i, k}^{c}-d_{i, j}^{u} \leq d_{i, k}^{c}-d_{i, j}^{u} \\
V_{i}^{c}-\min \left\{D_{i}^{u}, D_{i}^{c}\right\} \leq V_{i}^{c}-\min \left\{C_{i}^{u}, C_{i}^{c}\right\}
\end{gathered}
$$

Based on Equalities (2) and (3), we can get that $f_{i, k}^{c}-d_{i, j}^{u} \leq V_{i}^{c}-\min \left\{C_{i}^{u}, C_{i}^{c}\right\}$ if $d_{i, k}^{c}-d_{i, j}^{u} \leq$ $V_{i}^{c}-\min \left\{D_{i}^{u}, D_{i}^{c}\right\}$. Next, we prove $d_{i, k}^{c}-d_{i, j}^{u} \leq V_{i}^{c}-\min \left\{D_{i}^{u}, D_{i}^{c}\right\}$ by considering the following two cases.

- $d_{i, j}^{u}<d_{i, k-1}^{c}$. Since we consider a uni-processor system adopting the EDF scheduling, $J_{i, j}^{u}$ has a higher priority than $J_{i, k-1}^{c}$ in this case and $J_{i, k-1}^{c}$ cannot be executed in $\left[r_{i, j}^{u}, f_{i, j}^{u}\right)$. Since 


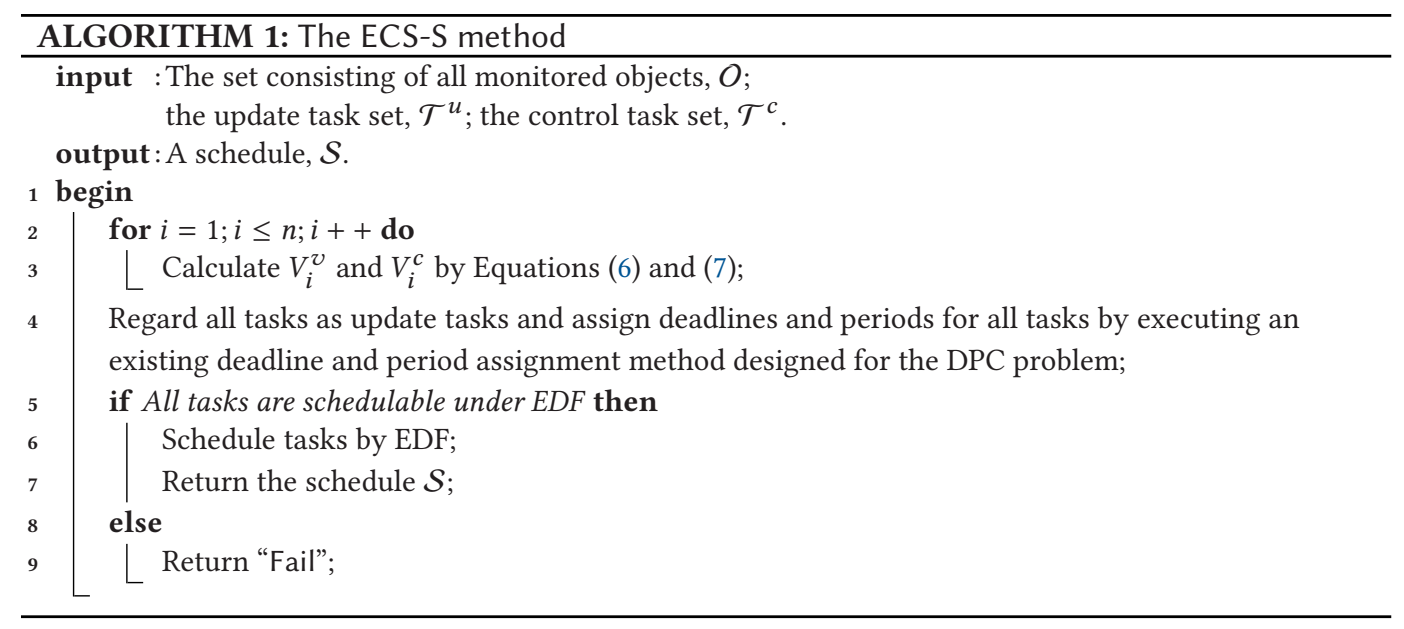

$J_{i, j}^{u}$ is the first job that captures the change, $d_{i}$ is updated by $J_{i, j}^{u}$ no later than $f_{i, j}^{u}$. Since control jobs read data no earlier than their start times and $J_{i, k-1}^{c}$ does not get the new $d_{i}$, the start time of $J_{i, k-1}^{c}$ must be earlier than the update time of $d_{i}$ by $J_{i, j}^{u}$ (also be earlier than the execution finish time of $J_{i, j}^{u}$ ), i.e., $s_{i, k-1}^{c}<f_{i, j}^{u}$. Note that $J_{i, k-1}^{c}$ cannot be executed in $\left[r_{i, j}^{u}, f_{i, j}^{u}\right)$. So, we have $s_{i, k-1}^{c}<r_{i, j}^{u}$. Since $r_{i, k-1}^{c} \leq s_{i, k-1}^{c}$, we can get the following equality:

$$
\begin{aligned}
d_{i, k}^{c}-d_{i, j}^{u} & =D_{i}^{c}+r_{i, k}^{c}-r_{i, j}^{u}-D_{i}^{u} \\
& =D_{i}^{c}+T_{i}^{c}+r_{i, k-1}^{c}-r_{i, j}^{u}-D_{i}^{u} \\
& =V_{i}^{c}+r_{i, k-1}^{c}-r_{i, j}^{u}-D_{i}^{u} \\
& \leq V_{i}^{c}+s_{i, k-1}^{c}-r_{i, j}^{u}-D_{i}^{u} \\
& <V_{i}^{c}-D_{i}^{u}
\end{aligned}
$$

- $d_{i, k-1}^{c} \leq d_{i, j}^{u}$. Since $V_{i}^{c}=T_{i}^{c}+D_{i}^{c}$, we can get that

$$
\begin{aligned}
d_{i, k}^{c}-d_{i, j}^{u} & =T_{i}^{c}+d_{i, k-1}^{c}-d_{i, j}^{u} \\
& \leq T_{i}^{c} \\
& \leq V_{i}^{c}-D_{i}^{u}
\end{aligned}
$$

Based on the above analysis, we can get that $d_{i, k}^{c}-d_{i, j}^{u} \leq V_{i}^{c}-\min \left\{D_{i}^{u}, D_{i}^{c}\right\}$, which means $f_{i, k}^{c}-d_{i, j}^{u} \leq$ $V_{i}^{c}-\min \left\{C_{i}^{u}, C_{i}^{c}\right\}$.

By $d_{i, j}^{u}-t<V_{i}^{u}$ and $f_{i, k}^{c}-d_{i, j}^{u} \leq V_{i}^{c}-\min \left\{C_{i}^{u}, C_{i}^{c}\right\}$, we can derive that $f_{i, k}^{c}-t=\left(f_{i, k}^{c}-d_{i, j}^{u}\right)+$ $\left(d_{i, j}^{u}-t\right)<V_{i}^{u}+V_{i}^{c}-\min \left\{C_{i}^{u}, C_{i}^{c}\right\}$, which means the theorem holds.

Theorem 4.1 gives an upper bound of the response time to each change of each monitored object. Based on Theorem 4.1, we can get that the system can respond to each change of each monitored object in time if all tasks are schedulable under EDF and $V_{i}^{u}+V_{i}^{c} \leq V_{i}+\min \left\{C_{i}^{u}, C_{i}^{c}\right\}$ for each task pair $\left\langle\tau_{i}^{u}, \tau_{i}^{c}>\right.$. Next, we consider how to assign reasonable values to $V_{i}^{u}$ and $V_{i}^{c}$. We notice that $T_{i}^{u}$ increases with the growth of $V_{i}^{u}$ under existing deadline and period assignment methods (designed for solving the DPC problem). Since $U_{i}^{u}=C_{i}^{u} / T_{i}^{u}$, the utilization of $\tau_{i}^{u}, U_{i}^{u}$, decreases with the growth of $V_{i}^{u}$. To get a smaller total utilization on premise that $V_{i}^{u}+V_{i}^{c} \leq V_{i}+\min \left\{C_{i}^{u}, C_{i}^{c}\right\}$, we let $V_{i}^{u}+V_{i}^{c}=V_{i}+\min \left\{C_{i}^{u}, C_{i}^{c}\right\}$. By considering the balance between $V_{i}^{c}$ and $V_{i}^{u}$, we set the 


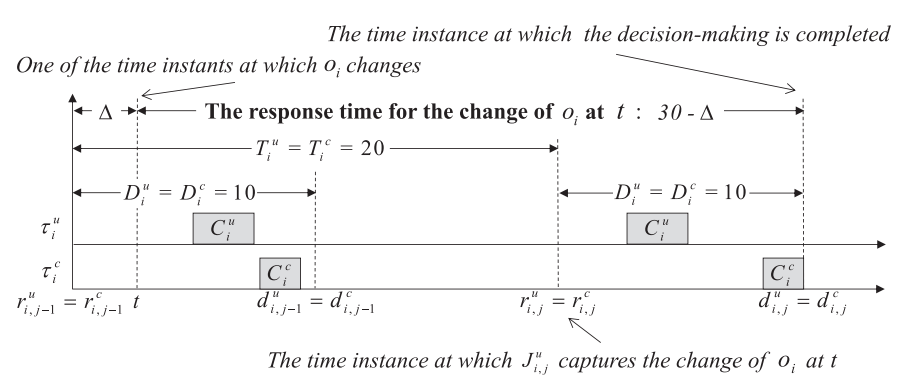

Fig. 1. A scenario in which the worst case response time for the change of a monitored object $o_{i}$ appears.

values of $V_{i}^{c}$ and $V_{i}^{u}$ by,

$$
\begin{gathered}
V_{i}^{c}=\left\lfloor\left(V_{i}+\min \left\{C_{i}^{u}, C_{i}^{c}\right\}\right) \cdot \frac{C_{i}^{c}}{C_{i}^{c}+C_{i}^{u}}\right\rfloor \\
V_{i}^{u}=V_{i}+\min \left\{C_{i}^{u}, C_{i}^{c}\right\}-V_{i}^{c}
\end{gathered}
$$

After obtaining $V_{i}^{c}$ and $V_{i}^{u}$, the deadline and period assignment for task pair $<\tau_{i}^{u}, \tau_{i}^{c}>$ can be realized by treating $\tau_{i}^{c}$ as an update task and executing an existing deadline and period assignment method (designed for the DPC problem) on $\tau_{i}^{u}$ and $\tau_{i}^{c}$. Based on the above analysis, we can get the ECS-S method as shown in Algorithm 1. Note that the timely response of the system to changes of monitored objects is realized on the premise that all tasks are schedulable under EDF. Therefore, we test the schedulability of each task after the deadline and period assignment.

Pessimism in ECS-S: In the proof of Theorem 4.1, we convert $f_{i, k}^{c}-t$ into $\left(f_{i, k}^{c}-d_{i, j}^{u}\right)+\left(d_{i, j}^{u}-t\right)$. In fact, $d_{i, j}^{u}-t$ is an upper bound of the time cost in the process of reflecting the change (at $t$ ) to the system, and the design idea of ECS-S is to divide the response process of the system to each change of each monitored object into two stages, the change reflection stage and the decisionmaking stage, and limit the time cost in each stage by assigning reasonable deadlines and periods for the corresponding update task and control task. Due to this division-based idea, ECS-S needs to leave enough space for the time cost in the decision-making stage and cannot assign a large value to each $V_{i}^{u}$. However, we find that a larger $V_{i}^{u}$ and a larger $V_{i}^{c}$ can be obtained if the deadlines and periods of $\tau_{i}^{u}$ and $\tau_{i}^{c}$ satisfy: (1) $D_{i}^{u}=D_{i}^{c}$; and (2) $T_{i}^{u}=T_{i}^{c}$. For example, given a monitored object $o_{i}$ with a valid response time $V_{i}=30$, if $C_{i}^{u}=C_{i}^{c}$, both $V_{i}^{u}$ and $V_{i}^{c}$ will be assigned to $V_{i} / 2=15$ under ECS-S. However, if we stipulate that update jobs have higher priorities when the absolute deadlines of update jobs and control jobs are the same, the response time of the system to each change of $o_{i}$ will not exceed $V_{i}=30$ when $D_{k}^{u}=D_{i}^{c}=10$ and $T_{i}^{u}=T_{i}^{c}=20$, since for each update job $J_{i, j}^{u}$, there is always a control job $J_{i, j}^{c}$ whose deadline is equal to the deadline of $J_{i, j}^{u}$ and release time is not earlier than the release time of $J_{i, j}^{u}$ (i,e., $J_{i, j}^{c}$ can get the new $d_{i}$ updated by $J_{i, j}^{u}$ and complete the decision-making before $d_{i, j}^{u}=d_{i, j}^{c}$ ). This means the response to each change of each monitored object can be completed before the absolute deadline of the first update job that captures this change. Figure 1 shows the scenario in which the worst case response time appears. Clearly, under the above settings, we can get a larger $V_{i}^{u}$ equal to $V_{i}^{u}=T_{i}^{u}+D_{i}^{u}=20+10=30>15$ and a larger $V_{i}^{c}$ equal to $V_{i}^{c}=T_{i}^{c}+D_{i}^{c}=20+10=30>15$. Based on the above idea, we develop a new method named HCS-S. Before showing our HCS-S method, we first give a theorem as follows.

THEOREM 4.2. For each monitored object ${ }_{i}$, if update jobs always have higher priorities than control jobs when the absolute deadlines of the update jobs and the control jobs are the same, and $\tau_{i}^{u}$ and $\tau_{i}^{c}$ satisfy: (1) $D_{i}^{u}=D_{i}^{c}$; (2) $T_{i}^{u}=T_{i}^{c}$; (3) both $\tau_{i}^{u}$ and $\tau_{i}^{c}$ are schedulable under EDF; and (4) $\tau_{i}^{u}$ and $\tau_{i}^{c}$ 


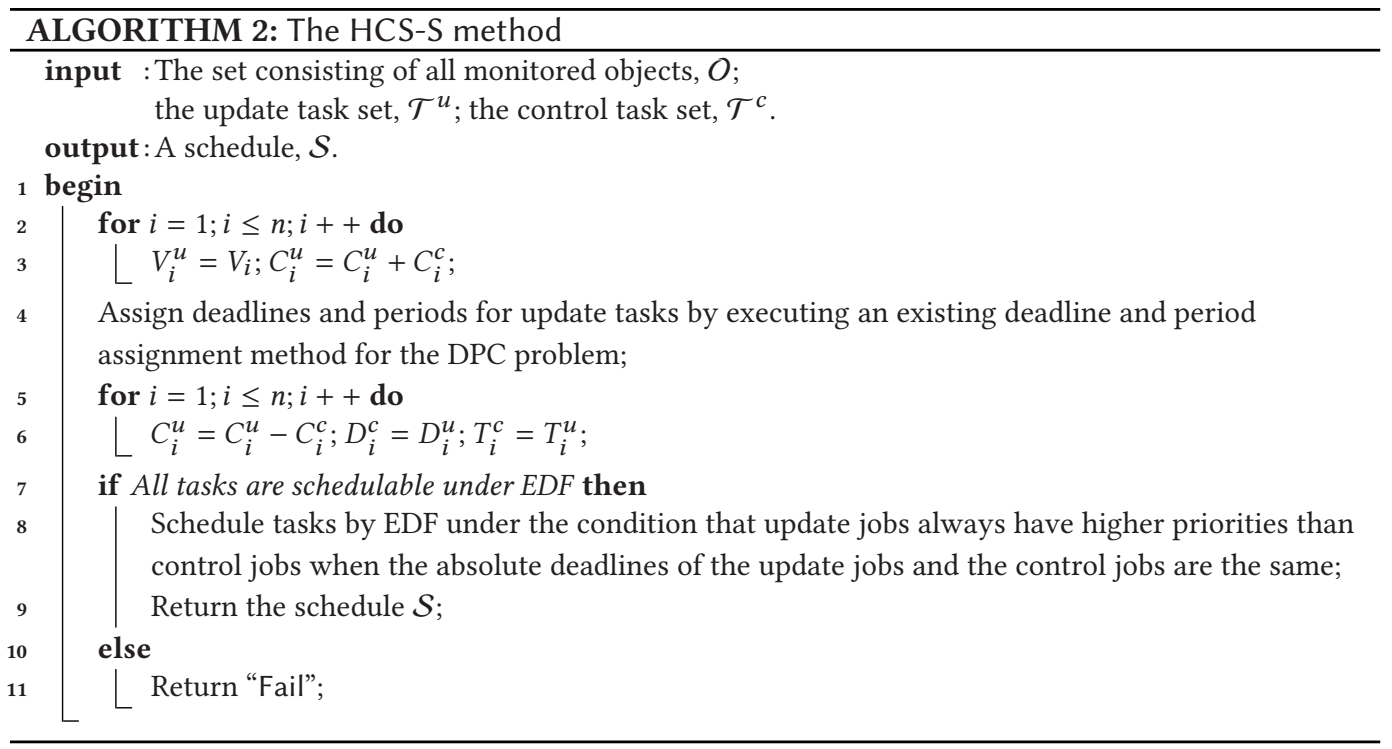

generate their first jobs at the same time, then the response time to each change of $o_{i}$ cannot exceed $D_{i}^{u}+T_{i}^{u}$.

Proof. Use $t$ to represent an arbitrary change time of $o_{i}$ and use $J_{i, j}^{u}$ to represent the first update job that captures this change. Since $T_{i}^{u}=T_{i}^{c}$ and $\tau_{i}^{u}$ and $\tau_{i}^{c}$ generate their first jobs at the same time, we can get that $r_{i, j}^{u}=r_{i, j}^{c}$. By $D_{i}^{u}=D_{i}^{c}$, we have $d_{i, j}^{u}=d_{i, j}^{c}$. Since update jobs always have higher priorities than control jobs when the absolute deadlines of the update jobs and the control jobs are the same, we can get that $J_{i, j}^{u}$ has a higher priority than $J_{i, j}^{c}$. Since $r_{i, j}^{u}=r_{i, j}^{c}, J_{i, j}^{c}$ cannot start its execution before the execution finish time of $J_{i, j}^{u}$, i.e., $f_{i, j}^{u} \leq s_{i, j}^{c}$. So, $J_{i, j}^{c}$ can get the new $d_{i}$ updated by $J_{i, j}^{u}$, which means the decision-making based on the change of $o_{i}$ at $t$ can be completed no later than $f_{i, j}^{c}$ and $f_{i, j}^{c}-t$ is an upper bound of the response time.

Next, we prove that $f_{i, j}^{c}-t<T_{i}^{u}+D_{i}^{u}$. Note that both $\tau_{i}^{u}$ and $\tau_{i}^{c}$ are schedulable. So, it can be obtained that $f_{i, j}^{c} \leq d_{i, j}^{c}$. Moreover, since $J_{i, j-1}^{u}$ does not capture the change of $o_{i}$ at $t$, the sample moment of $J_{i, j-1}^{u}$ must be earlier than $t$, i.e., $r_{i, j-1}^{u}<t$. By $f_{i, j}^{c} \leq d_{i, j}^{c}$ and $r_{i, j-1}^{u}<t$, we can get the following equality:

$$
\begin{aligned}
f_{i, j}^{c}-t & \leq d_{i, j}^{c}-t \\
& \leq d_{i, j}^{u}-t \\
& \leq D_{i}^{u}+T_{i}^{u}+r_{i, j-1}^{u}-t \\
& <D_{i}^{u}+T_{i}^{u}
\end{aligned}
$$

Since $f_{i, j}^{c}-t$ is an upper bound of the response time, the response time to the change of $o_{i}$ cannot exceed $D_{i}^{u}+T_{i}^{u}$.

Theorem 4.2 gives an upper bound of the response time. Based on Theorem 4.2, we can derive that the system can respond to each change of each monitored object in time if $T_{i}^{u}+D_{i}^{u}=V_{i}$ for each update task $\tau_{i}^{u}$ and all tasks satisfy the conditions in Theorem 4.2. Based on the above analysis, we can get the HCS-S method as shown in Algorithm 2. Clearly, HCS-S first sets each $V_{i}^{u}$ to $V_{i}$ and extends each $C_{i}^{u}$ to $C_{i}^{u}+C_{i}^{c}$ (at Lines 2 to 3). Then, the deadlines and periods of update tasks are assigned by executing an existing deadline and period assignment method (at Line 4). After 
obtaining the deadlines and periods of update tasks, HCS-S restores the WCET of each update task $\tau_{i}^{u}$ to $C_{i}^{u}$ and assigns deadline and period for each control task $\tau_{i}^{c}$ by $D_{i}^{c}=D_{i}^{u}$ and $T_{i}^{c}=T_{i}^{u}$ (at Lines 5 to 6). If all tasks are schedulable under EDF after the deadline and period assignment, HCS$\mathrm{S}$ uses EDF to schedule tasks under the condition that update jobs always have higher priorities than control jobs when the absolute deadlines of the update jobs and the control jobs are the same (at Line 8), and returns the final schedule $S$ (at Line 9). Otherwise, HCS-S returns "Fail" (at Line 11). Since we have not changed the priority assignment principle of the EDF scheduling, all existing schedulability analysis methods that support the EDF scheduling can be used in HCS-S.

\subsection{Methods in Complex Environments}

ECS-S and HCS-S are suitable for the environment where each control task accesses only one data item and each data item is accessed by only one control task. Next, we consider a complex environment where each data item may be accessed by multiple control tasks and each control task may access multiple data items, and propose two methods, ECS and HCS, to solve the DPC-RTC problem. Since each data item may be accessed by multiple control tasks, there may be multiple task pairs corresponding to each data item $d_{i}$. Since $\mathcal{T}_{i}^{c}$ is the task set consisting of all control tasks accessing $d_{i}$, we can get that following corollary based on Theorem 4.1.

Corollary 4.3. Let $V_{i}^{u}=D_{i}^{u}+T_{i}^{u}$ and $V_{k}^{c}=D_{k}^{c}+T_{k}^{c}$, if all tasks are schedulable under EDF, the response time of each change of each monitored object $o_{i}$ cannot exceed $\max \left\{V_{i}^{u}+V_{k}^{c}-\min \left\{C_{i}^{u}, C_{k}^{c}\right\} \mid \tau_{k}^{c} \in\right.$ $\mathcal{T}^{c}$.

Proof. Based on Theorem 4.1, we can get that the response time to each change of $o_{i}$ by each task pair $<\tau_{i}^{u}, \tau_{k}^{c}>$ with $\tau_{k}^{c}$ in $\mathcal{T}_{i}^{c}$ cannot exceed $V_{i}^{u}+V_{k}^{c}-\min \left\{C_{i}^{u}, C_{k}^{c}\right\}$. Since $\mathcal{T}_{i}^{c}$ consists of all control tasks accessing $d_{i}$, the response time to each change of $o_{i}$ cannot exceed $\max \left\{V_{i}^{u}+V_{k}^{c}-\right.$ $\left.\min \left\{C_{i}^{u}, C_{k}^{c}\right\} \mid \tau_{k}^{c} \in \mathcal{T}_{i}^{c}\right\}$.

Based on Corollary 4.3, we can get that the system can respond to each change of each monitored object $o_{i}$ in time if all tasks are schedulable under $\mathrm{EDF}$ and $\left.V_{i}^{u}+V_{k}^{c}-\min \left\{C_{i}^{u}, C_{k}^{c}\right\}\right\} \leq V_{i}$ for each task pair $<\tau_{i}^{u}, \tau_{k}^{c}>$ with $\tau_{k}^{c}$ in $\mathcal{T}_{i}^{c}$. By considering that a control task $\tau_{k}^{c}$ may access multiple data items, we change Equations (6) and (7) to Equations (9) and (10).

$$
\begin{gathered}
V_{k}^{c}=\min \left\{\left\lfloor\left(V_{i}+\min \left\{C_{i}^{u}, C_{k}^{c}\right\}\right) \cdot \frac{C_{k}^{c}}{C_{k}^{c}+C_{i}^{u}}\right\rfloor \mid d_{i} \in A_{k}^{c}\right\} \\
V_{i}^{u}=\min \left\{V_{i}+\min \left\{C_{i}^{u}, C_{k}^{c}\right\}-V_{k}^{c} \mid \tau_{k}^{c} \in \mathcal{T}_{i}^{c}\right\}
\end{gathered}
$$

Clearly, $V_{i}^{u}+V_{k}^{c}-\min \left\{C_{i}^{u}, C_{k}^{c}\right\} \leq V_{i}$ for each task pair $<\tau_{i}^{u}, \tau_{k}^{c}>$ with $\tau_{k}^{c}$ in $\mathcal{T}_{i}^{c}$ if all tasks satisfy Equations (9) and (10). Based on Corollary 4.3 and Equations (9) and (10), we can get the ECS method as shown in Algorithm 3. It should be pointed out that the execution results of ECS and ECS-S are the same in the simple environment introduced in Section 4.1, since there is only one task pair $\left\langle\tau_{i}^{u}, \tau_{k}^{c}>\right.$ that corresponds to $o_{i}$ (i.e., the calculation results of Equations (9) and (10) are equal to the calculation results of Equations (6) and (7), respectively).

Finally, we consider how to extend HCS-S to the complex environment. The reason why HCS-S can get some larger $V_{i}^{u}$ s and $V_{i}^{c}$ s is that, under HCS-S, the response to each change of each monitored object can be completed no later than the absolute deadline of the first update job that captures this change. To maintain the advantage of HCS-S, we let the extended HCS$\mathrm{S}$ method, HCS, inherit the above characteristic of HCS-S. We first consider the environment in which each data item may be accessed by multiple control tasks and each control task accesses only one data item. In this environment, each control task $\tau_{k}^{c}$ belongs to only one control task set 


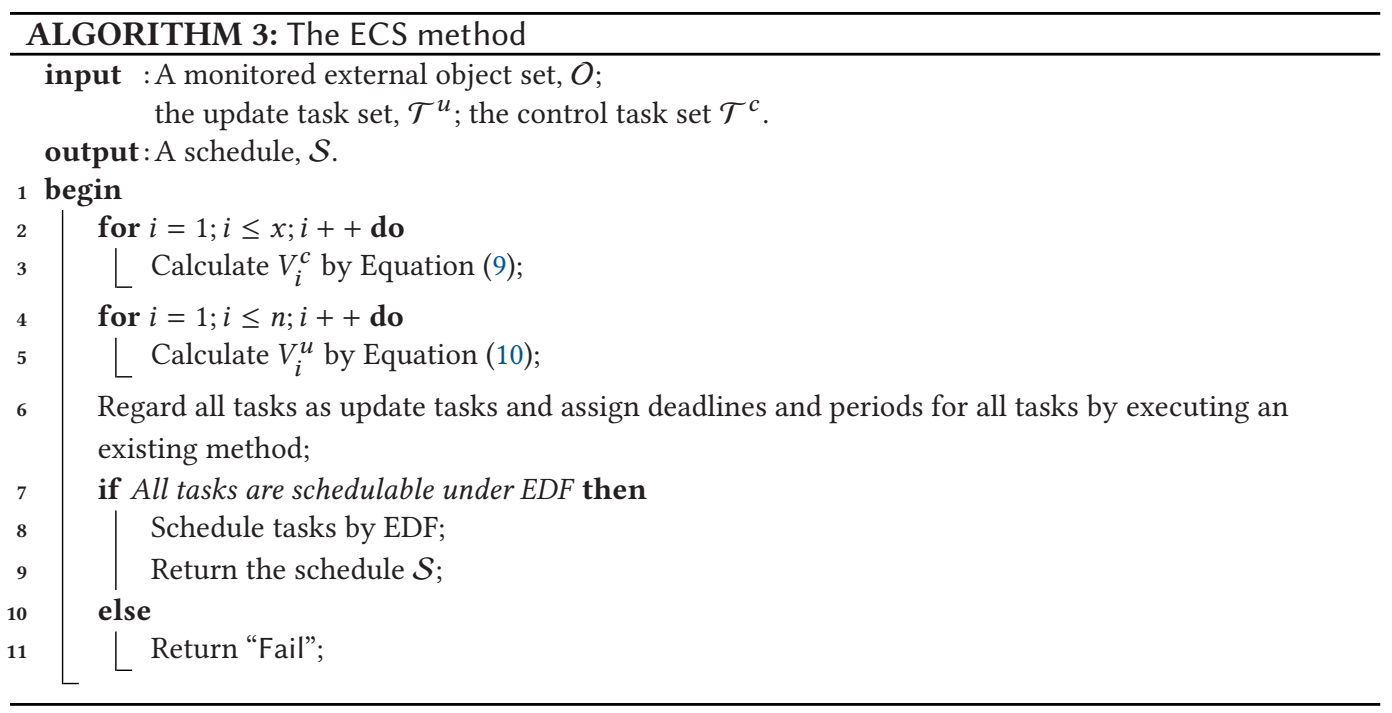

$\mathcal{T}_{i}^{c}$ that corresponds to the data item it accesses. Based on Theorem 4.2, we can get a corollary that holds in this environment.

Corollary 4.4. For each monitored object $o_{i}$, if update jobs always have higher priorities than control jobs when the absolute deadlines of the update jobs and the control jobs are the same, and the update task $\tau_{i}^{u}$ and all control tasks in $\mathcal{T}_{i}^{c}$ satisfy: (1) $D_{k}^{c}=D_{i}^{u}$ for each control task $\tau_{k}^{c}$ in $\mathcal{T}_{i}^{c}$; (2) $T_{k}^{c}=T_{i}^{u}$ for each control task $\tau_{k}^{c}$ in $\mathcal{T}_{i}^{c}$; (3) $\tau_{i}^{u}$ and all tasks in $\mathcal{T}_{i}^{c}$ are schedulable under EDF; and (4) $\tau_{i}^{u}$ and all control tasks in $\mathcal{T}_{i}^{c}$ generate their first jobs at the same time, then the response time to each change of $o_{i}$ cannot exceed $D_{i}^{u}+T_{i}^{u}$.

Proof. Based on Theorem 4.2, we can derive that the response time to each change of $o_{i}$ by each task pair $<\tau_{i}^{u}, \tau_{k}^{c}>$ with $\tau_{k}^{c}$ in $\mathcal{T}_{i}^{c}$ cannot exceed $D_{i}^{u}+T_{i}^{u}$ if $\tau_{i}^{u}$ and $\tau_{k}^{c}$ satisfy the conditions in Corollary 4.4. Since $\mathcal{T}_{i}^{c}$ consists of all control tasks accessing $d_{i}$ and all tasks in $\mathcal{T}_{i}^{c}$ satisfy the conditions in Corollary 4.4, the response time to each change of $o_{i}$ by each task pair corresponding to $d_{i}$ cannot exceed $D_{i}^{u}+T_{i}^{u}$, which means the response time to each change of $o_{i}$ cannot exceed $D_{i}^{u}+T_{i}^{u}$.

Based on Corollary 4.4, we can derive that, if we group tasks according to their corresponding data items, the HCS-S method can be used in the environment in which each data item may be accessed by multiple control tasks but each control task accesses only one data item. Next, we consider the environment in which each control task may access multiple data items. In this environment, a control task may correspond to multiple update tasks and the valid response times of the objects monitored by these update tasks may be different. So, the deadline and period assignment method in HCS-S is not suitable in this environment. However, we find that if the deadlines and periods of tasks satisfy some special relation, it still can be guaranteed that for each job $J_{i, j}^{u}$ of each update task $\tau_{i}^{u}$, every control task corresponding to $\tau_{i}^{u}$ (i.e., in $\mathcal{T}_{i}^{c}$ ) would generate a job with a release time no earlier than $r_{i, j}^{u}$ and an absolute deadline equal to $d_{i, j}^{u}$, which means the system can complete the response to the change captured by $J_{i, j}^{u}$ no later than $d_{i, j}^{u}$. The following theorem gives an upper bound of the response time when the deadlines and periods of tasks satisfy some special relation. 
THEOREM 4.5. For each monitored object $o_{i}$, if update jobs always have higher priorities than control jobs when the absolute deadlines of the update jobs and the control jobs are the same, and each task pair $<\tau_{i}^{u}, \tau_{k}^{c}>\left(\tau_{k}^{c} \in \mathcal{T}_{i}^{c}\right.$ ) satisfies: (1) $D_{k}^{c}=T_{k}^{c}$; (2) both $D_{i}^{u}$ and $T_{i}^{u}$ are integer multiples of $D_{k}^{c}$ (also $T_{k}^{c}$ ); (3) both $\tau_{i}^{u}$ and $\tau_{k}^{c}$ are schedulable under EDF; and (4) $\tau_{i}^{u}$ and $\tau_{k}^{c}$ generate their first jobs at the same time, then the response time to each change of $o_{i}$ cannot exceed $D_{i}^{u}+T_{i}^{u}$.

Proof. Use $t$ to represent an arbitrary change time of $o_{i}$ and use $J_{i, j}^{u}$ to represent the first job that captures this change. We first consider an arbitrary task pair $<\tau_{i}^{u}, \tau_{k}^{c}>$ with $\tau_{k}^{c}$ in $\mathcal{T}_{i}^{c}$ and prove that the response time for the change of $o_{i}$ at $t$ by $<\tau_{i}^{u}, \tau_{k}^{c}>$ cannot exceed $D_{i}^{u}+T_{i}^{u}$. Since $D_{k}^{c}=T_{k}^{c}$ and $D_{i}^{u}$ and $T_{i}^{u}$ are integer multiples of $D_{k}^{c}$, as well as $\tau_{i}^{u}$ and $\tau_{k}^{c}$ generate their first job at the same time, we can get that the absolute deadline of $J_{i, j}^{u}, d_{i, j}^{u}$, must be an integer multiple of $D_{k}^{c}$ and $T_{k}^{c}$, which means there must be a job $J_{k, l}^{c}$ that is generated by $\tau_{k}^{c}$ and has an absolute deadline equal to $d_{i, j}^{u}$ (i.e., $d_{k, l}^{c}=d_{i, j}^{u}$ ). Since $D_{i}^{u}$ is an integer multiple of $D_{k}^{c}$, we can get the following equality:

$$
\begin{aligned}
r_{k, l}^{c} & =d_{k, l}^{c}-D_{k}^{c} \\
& =d_{i, j}^{u}-D_{k}^{c} \\
& \geq d_{i, j}^{u}-D_{i}^{u} \\
& \geq r_{i, j}^{u}
\end{aligned}
$$

By $d_{k, l}^{c}=d_{i, j}^{u}$ and $r_{k, l}^{c} \geq r_{i, j}^{u}$, as well as the condition that update jobs always have higher priorities than control jobs when the absolute deadlines of the update jobs and the control jobs are the same, we can derive that $J_{k, l}^{c}$ can get the new $d_{i}$ updated by $J_{i, j}^{u}$ and the response to the change of $o_{i}$ at $t$ by $<\tau_{i}^{u}, \tau_{k}^{c}>$ will be completed no later than $f_{k, l}^{c}$. So, $f_{k, l}^{c}-t$ is an upper bound of the response time to this change by $\left\langle\tau_{i}^{u}, \tau_{k}^{c}>\right.$. Since $J_{i, j-1}^{u}$ does not capture this change, the release time of $J_{i, j-1}^{u}$ must be earlier than the change time $t$ (i.e., $r_{i, j-1}^{u}<t$ ). Since $\tau_{k}^{c}$ is schedulable, we have $f_{k, l}^{c} \leq d_{k, l}^{c}$. Therefore, the upper bound $f_{k, l}^{c}-t$ satisfies

$$
\begin{aligned}
f_{k, l}^{c}-t & \leq d_{k, l}^{c}-t \\
& \leq d_{i, j}^{u}-t \\
& \leq D_{i}^{u}+r_{i, j}^{u}-t \\
& \leq D_{i}^{u}+T_{i}^{u}+r_{i, j-1}^{u}-t \\
& <D_{i}^{u}+T_{i}^{u}
\end{aligned}
$$

This means the response time to the change of $o_{i}$ at $t$ by $<\tau_{i}^{u}, \tau_{k}^{c}>$ does not exceed $D_{i}^{u}+T_{i}^{u}$. Since $t$ is an arbitrary change time of $o_{i}$ and $\tau_{k}^{c}$ is an arbitrary control task in $\mathcal{T}_{i}^{c}$, the above conclusion holds for each change of $o_{i}$ and each task pair $\left\langle\tau_{i}^{u}, \tau_{k}^{c}>\right.$ with $\tau_{k}^{c}$ in $\mathcal{T}_{i}^{c}$. Therefore, the response time to each change of $o_{i}$ cannot exceed $T_{i}^{u}+D_{i}^{u}$.

Based on Theorem 4.5, we can get that the system can respond to the change of $o_{i}$ in time if $T_{i}^{u}+D_{i}^{u} \leq V_{i}$ and all task pairs $<\tau_{i}^{u}, \tau_{k}^{c}>\mathrm{s}\left(\tau_{k}^{c} \in \mathcal{T}_{i}^{c}\right)$ satisfy the conditions in Theorem 4.5. Note that there may be multiple tasks in $\mathcal{T}_{i}^{c}$. Since the deadline and period of $\tau_{i}^{u}$ need to be an integer multiple of the deadline of each task in $\mathcal{T}_{i}^{c}$ (to satisfy the conditions in Theorem 4.5), the deadlines of any two tasks in $\mathcal{T}_{i}^{c}$ need to satisfy the condition that the larger deadline is an integer multiple of the smaller deadline. Since the above condition can be satisfied if $\mathcal{T}_{i}^{c}$ is a harmonic task set, we can get the HCS method as shown in Algorithm 4.

HCS first initializes the $V_{i}^{u}$ of each update task $\tau_{i}^{u}$ to $V_{i}$ (in Lines 2 to 3). Then, HCS groups tasks according to their corresponding data items (at Line 4). More specifically, a graph is constructed by using nodes to represent tasks and connecting the nodes in each node pair that corresponds to 


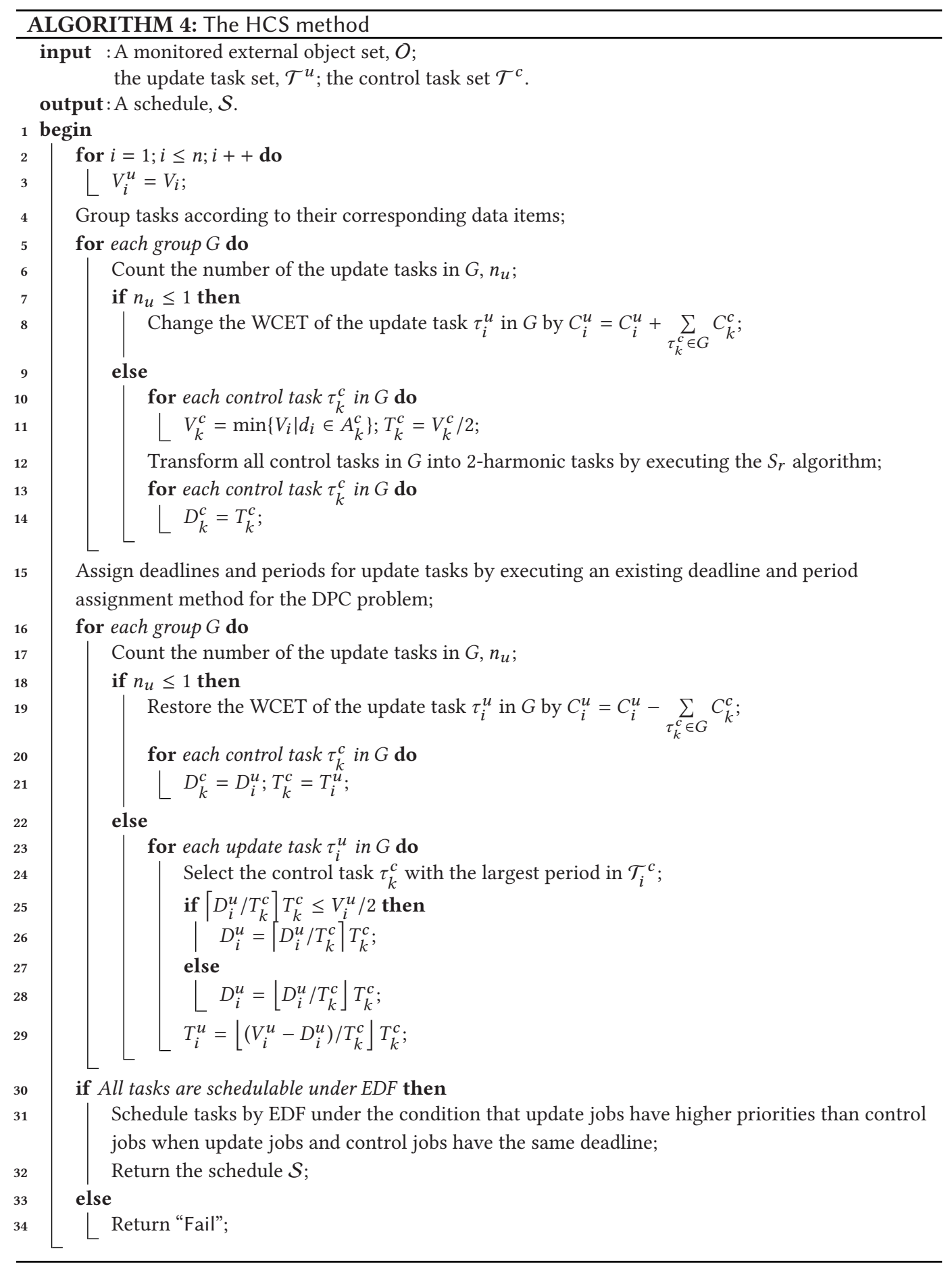




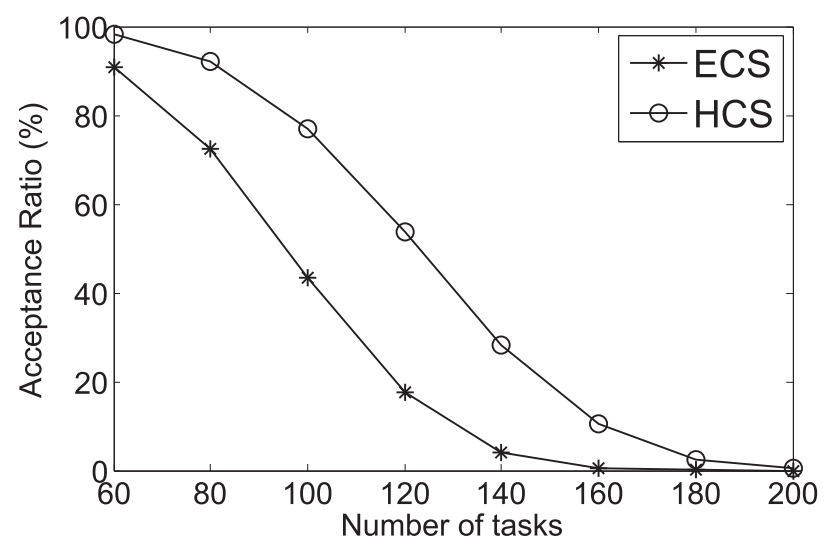

Fig. 2. Simple environments with $V=[100,10000]$ and $X=1$.

a task pair $\left\langle\tau_{i}^{u}, \tau_{k}^{c}>\right.$ satisfying $\tau_{k}^{c} \in \mathcal{T}_{i}^{c}$. The construction of the graph is the basis of the task grouping. Each group corresponds to a connected component in the graph. Next, for each group $G$, HCS counts the number of the update tasks in $G$ (at Line 6). If the number does not exceed 1, HCS uses a strategy similar to that in HCS-S to exceed the WCET of the update task $\tau_{i}^{u}$ in $G$ (at Line 8). Otherwise, for each control task $\tau_{k}^{c}$ in $G$, HCS initializes $V_{k}^{c}$ to $\min \left\{V_{i}^{u} \mid d_{i} \in A_{k}^{c}\right\}$ and initializes $T_{k}^{c}$ to $V_{k}^{c} / 2$ (at Line 11). Then, HCS transforms the task set consisting of all control tasks in $G$ into a 2-harmonic task set by executing the $S_{r}$ algorithm (that has been introduced in Section 3), and sets the deadline of each control task $\tau_{k}^{c}$ in $G$ to $T_{k}^{c}$. Next, HCS assigns the deadlines and periods to update tasks (at Line 15) and recounts the number of the update tasks in each group. If the number of the update tasks in a group $G$ does not exceed 1, HCS restores the WCET of the update task $\tau_{i}^{u}$ in $G$ (at Line 19), and sets the deadline (or period) of each control task in $G$ to the deadline (or period) of $\tau_{i}^{u}$ (in Lines 20 to 21). Clearly, after completing the above operation, all tasks in $G$ satisfy the conditions in Corollary 4.4. Otherwise, for each update task $\tau_{i}^{u}$ in $G$, HCS selects the control task $\tau_{k}^{c}$ with the largest deadline in $\mathcal{T}_{i}^{c}$ (at Line 24), and adjusts the deadline and period of $\tau_{i}^{u}$ to ensure the integer multiple relationship between the deadline (and period) of $\tau_{i}^{u}$ and the deadlines of the control tasks in $\mathcal{T}_{i}^{c}$ (in Lines 25 to 29). Clearly, after completing the above operation, all tasks in $G$ satisfy the conditions in Theorem 4.5. Finally, HCS tests the schedulability of all tasks by executing any existing schedulability analysis for tasks under the EDF schedule. If all tasks are schedulable, HCS schedules tasks by EDF under the condition that update jobs have higher priorities than control jobs when update tasks and control tasks have the same deadline (at Line 31), and returns the schedule (at Line 32). Otherwise, HCS returns "Fail" (at Line 34).

Note that in the simple environment, the execution results of ECS-S and ECS are the same, and the execution results of HCS-S and HCS are also the same. Among the above four methods, only ECS and HCS are suitable for the complex environment.

\section{EXPERIMENTAL EVALUATION}

In this section, we evaluate the performance of ECS and HCS in terms of acceptance ratio and execution efficiency, where acceptance ratio refers to the proportion of the schedulable tasks in the test task set. The MDC method [28] is used to assign deadlines and periods for tasks in ECS and HCS, and the QPA method [42] is used to test the schedulability of tasks in ECS and HCS. 


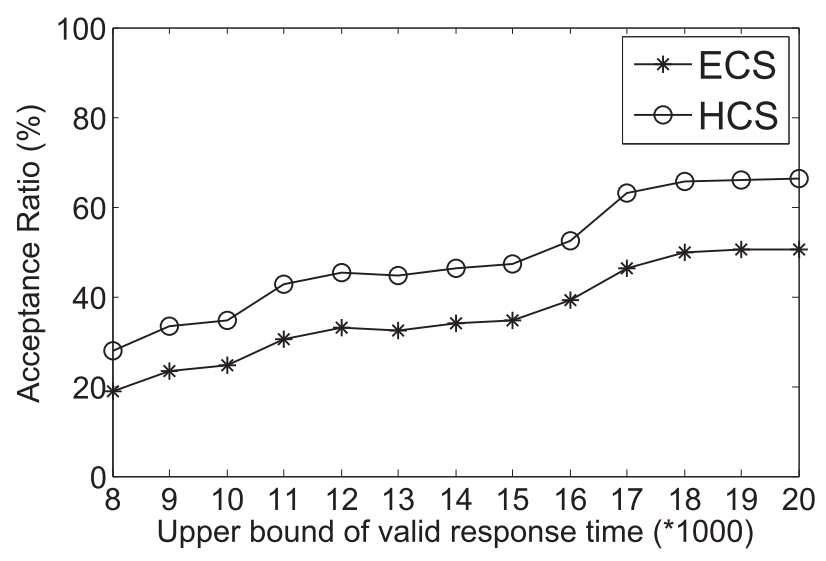

Fig. 3. Simple environments with $N=[30,300]$ and $X=1$.

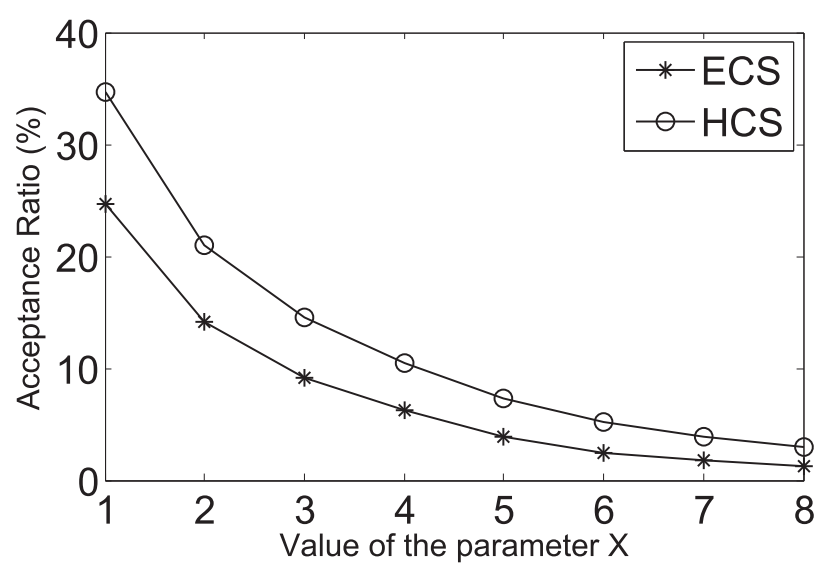

Fig. 4. Simple environments with $V=[100,10000]$ and $N=[30,300]$.

\subsection{Acceptance Ratio Test}

The parameter setting in our experiments is similar to that in [17] and [28]. More specifically, the default setting is as follows: the number of the tasks in each task set satisfies the uniform distribution in $N=[30,300]$; the WCET of each update task satisfies the uniform distribution in $[1,15]$; the WCET of each control task satisfies the uniform distribution in $[X, 15 X]$ (where $X$ is an integer with a default value equal to 1 ); the valid response time of each monitored object satisfies the uniform distribution in $V=[100,10000]$. In the simple environment, the number of update tasks (also the number of monitored objects) is equal to the number of control tasks. In the complex environment, update tasks account for $P=60 \%$ of all tasks, the number of the data items accessed by each control task satisfies the uniform distribution in $[1,3]$, and the data items accessed by each control task are assigned randomly. 10000 task sets are conducted in our experiment. Figures 2 to 8 show the comparison between ECS and HCS. The following trends can be derived from the comparison:

1. As shown in Figures 2 and 5, the acceptance ratios of both HCS and ECS decrease with the growth of the number of tasks. HCS has a better acceptance ratio than ECS in both the simple 


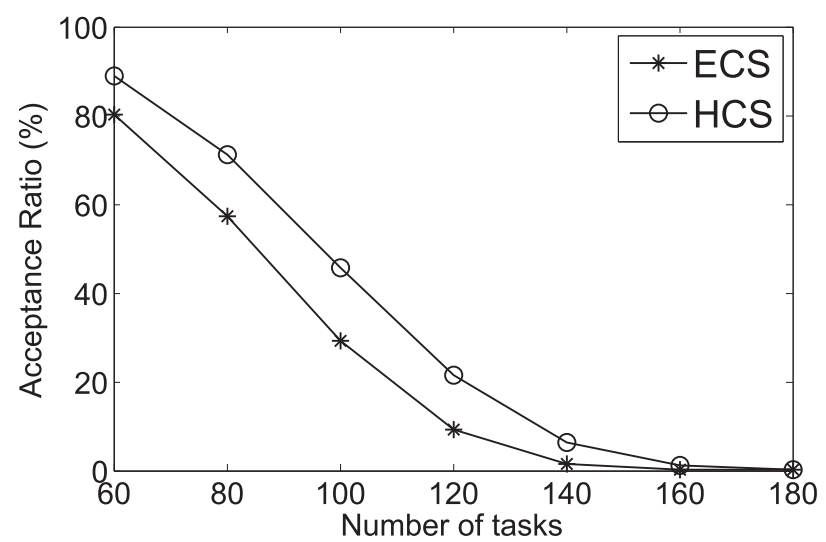

Fig. 5. Complex environments with $P=60 \%, V=[100,10000]$ and $X=1$.

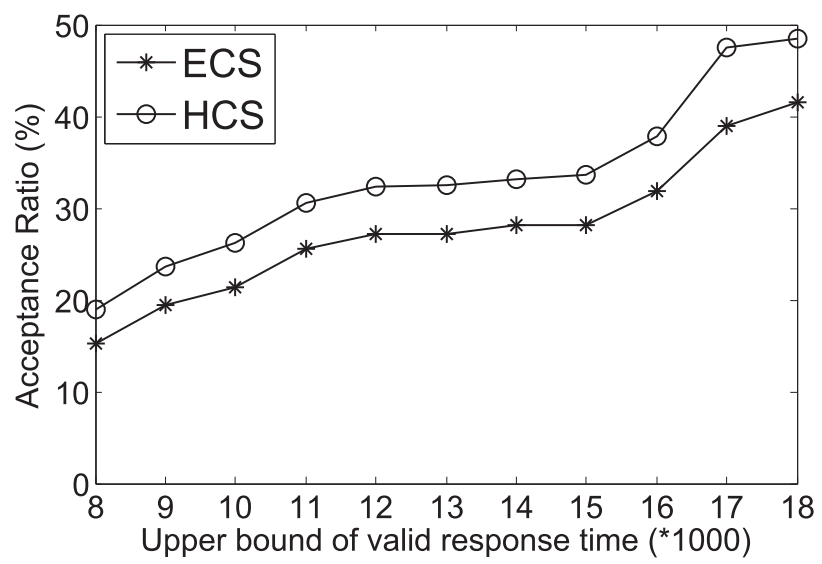

Fig. 6. Complex environments with $P=60 \%, N=[30,300]$ and $X=1$.

environment and the complex environment. In the simple environment, the absolute difference between the acceptance ratios of HCS and ECS can reach about 36.5\% when the number of tasks is equal to 120. In the complex environment, the absolute difference between the acceptance ratios of HCS and ECS can reach about $18 \%$ when the total number of tasks is equal to 100 .

2. Since the total utilization of the task set decreases with the growth of the upper bound of $V$, the acceptance ratios of both HCS and ECS increase with the growth of the upper bound of $V$ (as shown in Figures 3 and 6). In the simple environment, the average acceptance ratios of HCS and ECS are about $49.1 \%$ and $33.9 \%$, respectively. When the upper bound of $V$ is equal to 8000 , the acceptance ratio of HCS is about 1.5 times larger than that of ECS. In the complex environment, the average acceptance ratios of HCS and ECS are about $35.2 \%$ and $29.7 \%$, respectively. When the upper bound of $V$ is equal to 9000 , the acceptance ratio of HCS is about 1.25 times larger than that of ECS.

3. As shown in Figure 7, in the complex environment, the acceptance ratios of both HCS and ECS increase with the growth of the proportion of update tasks in the task set. The absolute difference between the acceptance rates of the above two methods is almost unaffected by the size of the 


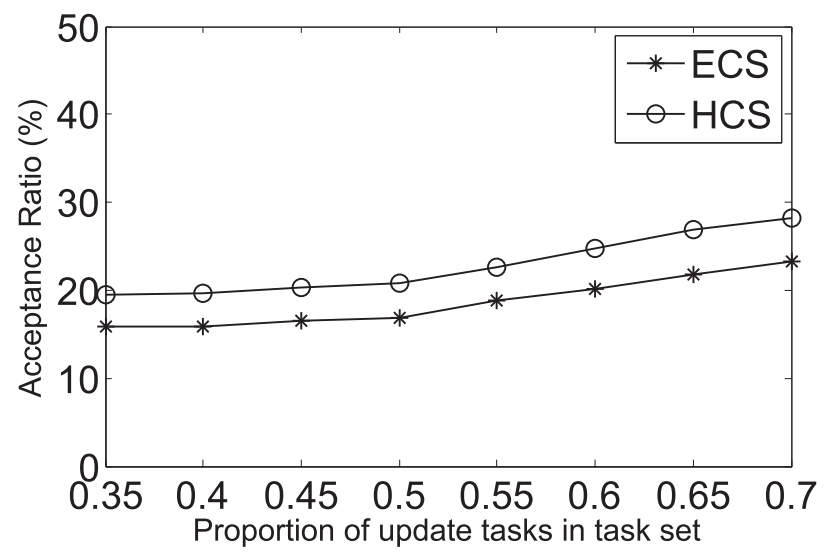

Fig. 7. Complex environments with $V=[100,10000], N=[30,300]$ and $X=1$.

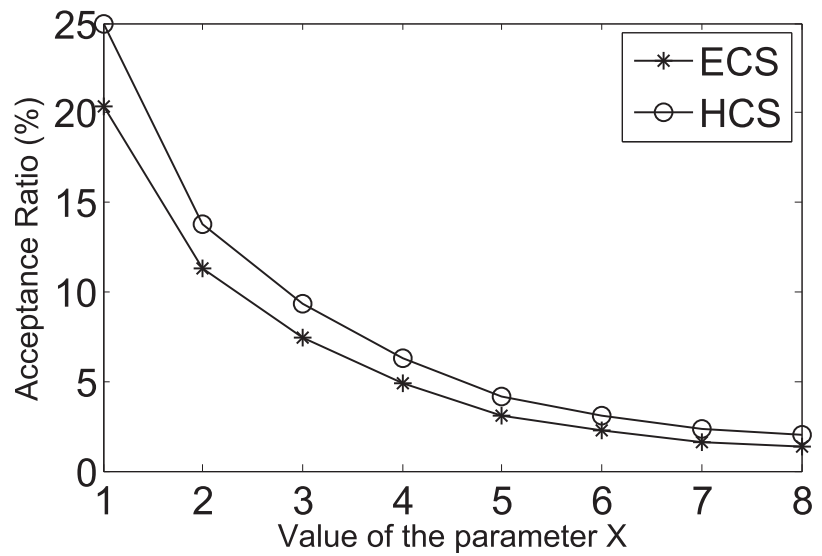

Fig. 8. Complex environments with $P=60 \%, V=[100,10000]$ and $N=[30,300]$.

proportion. When the proportion is equal to $65 \%$, the acceptance ratio of HCS is about 1.25 times larger than that of ECS.

4. As shown in Figures 4 and 8, the acceptance ratios of both HCS and ECS decrease with the growth of $X$ in both the simple environment and the complex environment. The acceptance ratio of HCS is on average 1.7 times larger than that of ECS. The acceptance ratio of HCS is more than twice that of ECS at $X=8$ in the simple environment. The acceptance ratio of HCS is about 1.5 times that of ECS at $X=8$ in the complex environment.

5. As shown in Figures 2 to 8, the acceptance ratio of HCS is always better than that of ECS in both the simple environment and the complex environment. Compared with that in the complex environment, the advantage of HCS over ECS is more obvious in the simple environment.

\subsection{Execution Efficiency Test}

A system with an Intel(R) Core(TM) $i 5-7300 \mathrm{U} 8 \mathrm{G} 2.60 \mathrm{GHz}$ CPU is used to test the execution efficiency of the proposed methods. The default parameter settings in this experiment are the 
Table 2. Average Runtime in the Simple Environment (ms)

\begin{tabular}{rrr|crc|crr}
\hline \multicolumn{1}{r}{$\mathrm{N}$} & \multicolumn{1}{c}{ ECS } & HCS & V & \multicolumn{1}{c|}{ ECS } & \multicolumn{1}{c|}{ HCS } & X & \multicolumn{1}{c}{ ECS } & \multicolumn{1}{c}{ HCS } \\
\hline 60 & 247.81 & 123.97 & {$[100,8000]$} & 32.55 & 318.85 & 1 & 58.64 & 425.54 \\
80 & 240.03 & 183.99 & {$[100,10000]$} & 58.64 & 425.54 & 2 & 36.17 & 371.18 \\
100 & 238.69 & 206.02 & {$[100,12000]$} & 136.23 & 676.96 & 3 & 18.17 & 150.05 \\
120 & 125.84 & 231.45 & {$[100,14000]$} & 143.45 & 731.08 & 4 & 7.04 & 76.62 \\
140 & 77.75 & 282.53 & {$[100,16000]$} & 211.47 & 907.47 & 5 & 4.14 & 49.39 \\
160 & 44.15 & 368.13 & {$[100,18000]$} & 488.74 & 1009.43 & 6 & 3.55 & 32.56 \\
180 & 27.54 & 462.27 & {$[100,20000]$} & 561.81 & 1027.48 & 7 & 2.37 & 24.68 \\
200 & 19.29 & 564.27 & & & & 8 & 1.91 & 16.29 \\
\hline
\end{tabular}

Table 3. Average Runtime in the Complex Environment (ms)

\begin{tabular}{rrr|crc|ccc|ccc}
\hline \multicolumn{1}{r|}{ N } & \multicolumn{1}{c|}{ ECS } & \multicolumn{1}{c|}{ HCS } & V & \multicolumn{1}{c|}{ ECS } & \multicolumn{1}{c}{ HCS } & P & ECS & HCS & X & ECS & \multicolumn{1}{c}{ HCS } \\
\hline 60 & 140.72 & 145.25 & {$[100,8000]$} & 40.93 & 538.09 & $35 \%$ & 22.28 & 160.15 & 1 & 59.67 & 608.13 \\
80 & 154.14 & 210.24 & {$[100,10000]$} & 59.67 & 608.13 & $40 \%$ & 25.61 & 226.97 & 2 & 18.52 & 185.32 \\
100 & 135.06 & 520.05 & {$[100,12000]$} & 96.56 & 972.05 & $45 \%$ & 32.83 & 352.18 & 3 & 10.01 & 92.46 \\
120 & 109.48 & 741.91 & {$[100,14000]$} & 105.74 & 1032.13 & $50 \%$ & 37.08 & 430.91 & 4 & 5.77 & 49.12 \\
140 & 58.17 & 888.07 & {$[100,16000]$} & 162.04 & 1442.88 & $55 \%$ & 40.16 & 509.12 & 5 & 4.19 & 29.98 \\
160 & 26.36 & 952.81 & {$[100,18000]$} & 348.75 & 1786.47 & $60 \%$ & 59.67 & 608.13 & 6 & 2.27 & 23.25 \\
180 & 12.59 & 1003.69 & {$[100,20000]$} & 339.02 & 1933.38 & $65 \%$ & 63.35 & 815.25 & 7 & 1.99 & 16.62 \\
200 & 16.13 & 1496.74 & & & & $70 \%$ & 87.75 & 904.83 & 8 & 1.76 & 11.74 \\
\hline
\end{tabular}

same as that in the acceptance ratio experiment. The performances of ECS and HCS are shown in Tables 2 to 3. The following trends can be derived from the comparison:

1. The average runtime of ECS decreases with the growth of the number of tasks, while average runtime of HCS increases with the growth of the number of tasks. The reason is that the acceptance ratio of ECS decreases with the growth of the number of tasks and the schedulability analysis method (i.e., MDC) used in ECS can terminate the execution of ECS once there appears an unschedulable task. However, HCS needs to complete the task transformation (by executing the $S_{r}$ method) before the schedulability analysis and the runtime of $S_{r}$ increases with the growth of the number of tasks. Therefore, although the MDC method (executed in HCS) can terminate the execution of HCS once there appears an unschedulable task, the average runtime of HCS increases with the growth of the number of tasks. The execution efficiency of ECS is higher than that of HCS in the complex environment. But, in the simple environment, the execution efficiency of HCS is higher than that of ECS when the number of tasks does not exceed 100, and the execution efficiency of ECS is higher than that of HCS when the number of tasks is no less than 120. The average runtime of ECS is about $3.4 \%$ (or 1.1\%) of the average runtime of HCS at $N=200$ in the simple environment (or in the complex environment).

2. Since the valid response times of tasks (i.e., $V_{i}^{c} s$ and $V_{i}^{u} s$ ) increase with the growth of the upper bound of $V$ and the runtime of the schedulability analysis method (used in both ECS and HCS) increases with the growth of the valid response time of tasks, the average runtime of ECS and HCS increases with the growth of the upper bound of $V$. ECS always has a better performance than HCS in both the simple environment and the complex environment. The ratio between the average runtime of ECS and HCS increases with the growth of the upper bound of $V$.

3. The average runtime of both ECS and HCS increases with the growth of the proportion of update tasks in the task set. The ratio between the average runtime of ECS and HCS decreases 
with the growth of the proportion of update tasks in the task set. When the proportion is equal to $35 \%$, the average runtime of ECS and HCS is $22.28 \mathrm{~ms}$ and $160.15 \mathrm{~ms}$, respectively, and the ratio is about $1: 7.2$. When the proportion is equal to $70 \%$, the average runtime of ECS and HCS is $87.75 \mathrm{~ms}$ and $904.83 \mathrm{~ms}$, respectively, and the ratio is about $1: 10.3$.

4. The average runtime of both ECS and HCS decreases with the growth of $X$ in both the simple environment and the complex environment. The average runtime of ECS is about $10.5 \%$ of the average runtime of HCS in the simple environment. The average runtime of ECS is about $11.5 \%$ of the average runtime of HCS in the complex environment.

5. The execution efficiency of ECS is higher than that of HCS in most cases (excluding the case in which the number of tasks are less than 120 in the sample environment). When the number of tasks does not exceed 160 and the upper bound of $V$ does not exceed 12000, the average runtime of both ECS and HCS does not exceed 1s.

\section{CONCLUSIONS}

To the best of our knowledge, this is the first work that focuses on the deadline and period assignment for both update tasks and control tasks, with the goal of ensuring timely response to the changes of monitored objects. A new problem about the response time control and the system workload control is defined in this paper. Two deadline and period assignment methods are proposed to solve the defined problem. Experiments with randomly generated tasks are conducted to evaluate the performance of the proposed methods in terms of acceptance ratio and execution efficiency.

\section{REFERENCES}

[1] Matthias Becker, Dakshina Dasari, Saad Mubeen, Moris Behnam, and Thomas Nolte. 2016. Synthesizing job-level dependencies for automotive multi-rate effect chains. In 2016 IEEE 22nd International Conference on Embedded and Real-Time Computing Systems and Applications (RTCSA). IEEE, 159-169.

[2] Matthias Becker, Dakshina Dasari, Saad Mubeen, Moris Behnam, and Thomas Nolte. 2017. End-to-end timing analysis of cause-effect chains in automotive embedded systems. fournal of Systems Architecture 80 (2017), 104-113.

[3] Alessandro Biondi and Marco Di Natale. 2018. Achieving predictable multicore execution of automotive applications using the let paradigm. In 2018 IEEE Real-Time and Embedded Technology and Applications Symposium (RTAS). IEEE, $240-250$

[4] Mokrane Bouzeghoub. 2004. A framework for analysis of data freshness. In Proceedings of the 2004 international workshop on Information quality in information systems. 59-67.

[5] Nico Feiertag, Kai Richter, Johan Nordlander, and Jan Jonsson. 2009. A compositional framework for end-to-end path delay calculation of automotive systems under different path semantics. In IEEE Real-Time Systems Symposium: 30/11/2009-03/12/2009. IEEE Communications Society.

[6] Chenchen Fu, Qiangqiang Liu, Peng Wu, Minming Li, Chun Jason Xue, Yingchao Zhao, Jingtong Hu, and Song Han. 2018. Real-time data retrieval in cyber-physical systems with temporal validity and data availability constraints. IEEE Transactions on Knowledge and Data Engineering 31, 9 (2018), 1779-1793.

[7] Chenchen Fu, Peng Wu, Minming Li, Chun Jason Xue, Yingchao Zhao, and Song Han. 2018. Real-time data retrieval with multiple availability intervals in CPS under freshness constraints. IEEE Transactions on Computer-Aided Design of Integrated Circuits and Systems 37, 11 (2018), 2743-2754.

[8] Richard Gerber, Seongsoo Hong, and Manas Saksena. 1994. Guaranteeing end-to-end timing constraints by calibrating intermediate processes. In IEEE Real-Time Systems Symposium. IEEE, 192-203.

[9] Dagaen Golomb, Deepak Gangadharan, Sanjian Chen, Oleg Sokolsky, and Insup Lee. 2018. Data freshness overengineering: Formulation and results. In 2018 IEEE 21st International Symposium on Real-Time Distributed Computing (ISORC). IEEE, 174-183.

[10] Thomas Gustafsson and Jörgen Hansson. 2004. Data management in real-time systems: A case of on-demand updates in vehicle control systems. In IEEE Real-Time and Embedded Technology and Applications Symposium. IEEE, 182-191.

[11] Thomas Gustafsson and Jörgen Hansson. 2004. Dynamic on-demand updating of data in real-time database systems. In ACM symposium on Applied computing. 846-853.

[12] Ching-Chih Han, Kwei-Jay Lin, and Chao-Ju Hou. 1996. Distance-constrained scheduling and its applications to realtime systems. IEEE Transactions on computers 45, 7 (1996), 814-826. 
[13] Song Han, Deji Chen, Ming Xiong, Kam-yiu Lam, Aloysius K. Mok, and Krithi Ramamritham. 2012. Schedulability analysis of deferrablescheduling algorithms for maintainingreal-time data freshness. IEEE Trans. Comput. 63, 4 (2012), 979-994.

[14] Song Han, Deji Chen, Ming Xiong, Kam-yiu Lam, Aloysius Ka-Lau Mok, and Krithi Ramamritham. 2012. Schedulability analysis of deferrablescheduling algorithms for maintainingreal-time data freshness. IEEE Trans. Comput. 63, 4 (2012) 979-994.

[15] Song Han, Deji Chen, Ming Xiong, and Aloysius Ka-Lau Mok. 2009. Online scheduling switch for maintaining data freshness in flexible real-time systems. In IEEE Real-Time Systems Symposium. IEEE, 115-124.

[16] Song Han, Kam-Yiu Lam, Deji Chen, Ming Xiong, Jiantao Wang, Krithi Ramamritham, and Aloysius K. Mok. 2015. Online mode switch algorithms for maintaining data freshness in dynamic cyber-physical systems. IEEE Transactions on Knowledge and Data Engineering 28, 3 (2015), 756-769.

[17] Song Han, Kam-Yiu Lam, Jiantao Wang, Krithi Ramamritham, and Aloysius Ka-Lau Mok. 2012. On co-scheduling of update and control transactions in real-time sensing and control systems: Algorithms, analysis, and performance IEEE Transactions on Knowledge and Data Engineering 25, 10 (2012), 2325-2342.

[18] Song Han, Kam-yiu Lam, Jiantao Wang, Sang Hyuk Son, and Aloysius Ka-Lau Mok. 2012. Adaptive co-scheduling for periodic application and update transactions in real-time database systems. fournal of Systems and Software 85, 8 (2012), 1729-1743.

[19] Song Han, Aloysius Ka-Lau Mok, Jianyong Meng, Yi-Hung Wei, Pei-Chi Huang, Quan Leng, Xiuming Zhu, Luis Sentis, Kwan Suk Kim, and Risto Miikkulainen. 2013. Architecture of a cyberphysical avatar. In ACM/IEEE International Conference on Cyber-Physical Systems. 189-198.

[20] Shao-Juen Ho, Tei-Wei Kuo, and Aloysius Ka-Lau Mok. 1997. Similarity-based load adjustment for real-time dataintensive applications. In IEEE Real-Time Systems Symposium. IEEE, 144-153.

[21] Abhay Kumar Jha, Ming Xiong, and Krithi Ramamritham. 2006. Mutual consistency in real-time databases. In IEEE Real-Time Systems Symposium. IEEE, 335-343.

[22] Kyoung-Don Kang, Sang Hyuk Son, John Stankovic, and Tarek F. Abdelzaher. 2002. A QoS-sensitive approach for timeliness and freshness guarantees in real-time databases. In Euromicro Conference on Real-Time Systems. IEEE, 203212.

[23] Young-Kuk Kim and Sang Hyuk Son. 1995. Predictability and consistency in real-time database systems. Advances in real-time systems (1995), 509-531.

[24] JeongGil Ko, Chenyang Lu, Mani B. Srivastava, John Stankovic, Andreas Terzis, and Matt Welsh. 2010. Wireless sensor networks for healthcare. Proc. IEEE 98, 11 (2010), 1947-1960

[25] Tei-Wei Kuo and Shao-Juen Ho. 2000. Similarity-based load adjustment for static real-time transaction systems. IEEE Trans. Comput. 49, 2 (2000), 112-126.

[26] Tei-Wei Kuo and Aloysius Ka-Lau Mok. 1993. SSP: A semantics-based protocol for real-time data access. In IEEE Real-Time Systems Symposium. IEEE, 76-86.

[27] Kam-Yiu Lam, Ming Xiong, BiYu Liang, and Yang Guo. 2004. Statistical quality of service guarantee for temporal consistency of real-time data objects. In IEEE Real-Time Systems Symposium. IEEE, 276-285.

[28] Guohui Li, Chenggang Deng, Jianjun Li, Quan Zhou, and Wei Wei. 2016. Deadline and period assignment for update transactions in co-scheduling environment. IEEE Trans. Comput. 66, 7 (2016), 1119-1131.

[29] Guohui Li, Jianjun Li, Bing Guo, et al. 2019. Maintaining data freshness in distributed cyber-physical systems. IEEE Trans. Comput. 68, 7 (2019), 1077-1090.

[30] Jianjun Li, Jian-Jia Chen, Ming Xiong, and Guohui Li. 2011. Workload-aware partitioning for maintaining temporal consistency upon multiprocessor platforms. In IEEE Real-Time Systems Symposium. IEEE, 126-135.

[31] Jianjun Li, Jian-Jia Chen, Ming Xiong, Guohui Li, and Wei Wei. 2015. Temporal consistency maintenance upon partitioned multiprocessor platforms. IEEE Trans. Comput. 65, 5 (2015), 1632-1645.

[32] Chenyang Lu, Abusayeed Saifullah, Bo Li, Mo Sha, Humberto Gonzalez, Dolvara Gunatilaka, Chengjie Wu, Lanshun Nie, and Yixin Chen. 2015. Real-time wireless sensor-actuator networks for industrial cyber-physical systems. Proc. IEEE 104, 5 (2015), 1013-1024.

[33] Verónika Peralta. 2006. Data freshness and data accuracy: A state of the art. Instituto de Computacion, Facultad de Ingenieria, Universidad de la Republica2006 (2006).

[34] Xuesen Shi, Yuyao Shen, Yongqing Wang, and Li Bai. 2018. Differential-clustering compression algorithm for real-time aerospace telemetry data. IEEE Access 6 (2018), 57425-57433.

[35] Xiaohui Song and Jane W.-S. Liu. 1995. Maintaining temporal consistency: Pessimistic vs. optimistic concurrency control. IEEE transactions on knowledge and data engineering 7, 5 (1995), 786-796.

[36] Jiantao Wang, Song Han, Kam-Yiu Lam, and Aloysius Ka-Lau Mok. 2012. Maintaining data temporal consistency in distributed real-time systems. Real-Time Systems 48, 4 (2012), 387-429. 
[37] Jian-Tao Wang, Kam-Yiu Lam, Song Han, Sang Hyuk Son, and Aloysius Ka-Lau Mok. 2013. An effective fixed priority co-scheduling algorithm for periodic update and application transactions. Computing 95, 10 (2013), 993-1018.

[38] Ming Xiong, Song Han, and Kam-Yiu Lam. 2005. A deferrable scheduling algorithm for real-time transactions maintaining data freshness. In IEEE Real-Time Systems Symposium. IEEE, 11-pp.

[39] Ming Xiong, Song Han, Kam-Yiu Lam, and Deji Chen. 2008. Deferrable scheduling for maintaining real-time data freshness: Algorithms, analysis, and results. IEEE Trans. Comput. 57, 7 (2008), 952-964.

[40] Ming Xiong and Krithi Ramamritham. 2004. Deriving deadlines and periods for real-time update transactions. IEEE Trans. Comput. 53, 5 (2004), 567-583.

[41] Ming Xiong, Krithi Ramamritham, John Stankovic, Don Towsley, and Rajendran Sivasankaran. 2002. Scheduling transactions with temporal constraints: Exploiting data semantics. IEEE transactions on knowledge and Data Engineering 14, 5 (2002), 1155-1166.

[42] Fengxiang Zhang and Alan Burns. 2009. Schedulability analysis for real-time systems with EDF scheduling. IEEE Trans. Comput. 58, 9 (2009), 1250-1258.

[43] Fangzhou Zhu, Jianjun Li, and Guohui Li. 2011. An efficient deadline and period assignment scheme for maintaining temporal consistency under edf. In International Conference on Human-centric Computing and Embedded and Multimedia Computing. Springer, 351-364.

Received April 2021; revised May 2021; accepted July 2021 